\title{
Production and Decay of Up-Type and Down-Type New Heavy Quarks through Anomalous Interactions at the LHC
}

\author{
İ. T. Çakur, ${ }^{1}$ S. Kuday, ${ }^{1}$ and O. Çakur ${ }^{1,2}$ \\ ${ }^{1}$ Application and Research Center for Advanced Studies, Istanbul Aydin University, Sefakoy, 34295 Istanbul, Turkey \\ ${ }^{2}$ Department of Physics, Ankara University, Tandogan, 06100 Ankara, Turkey
}

Correspondence should be addressed to İ. T. Çakır; ilkay.turk.cakir@cern.ch

Received 23 October 2014; Revised 19 January 2015; Accepted 26 January 2015

Academic Editor: Hong-Jian He

Copyright ( 2015 İ. T. Çakır et al. This is an open access article distributed under the Creative Commons Attribution License, which permits unrestricted use, distribution, and reproduction in any medium, provided the original work is properly cited. The publication of this article was funded by SCOAP $^{3}$.

\begin{abstract}
We study the process $p p \rightarrow Q V+X$ (where $Q=t, b$ and $V=g, \gamma$, and $Z$ ) through the anomalous interactions of the new heavy quarks at the LHC. Considering the present limits on the masses and mixings, the signatures of the heavy quark anomalous interactions are discussed and analysed at the LHC for the center of mass energy of $13 \mathrm{TeV}$. An important sensitivity to anomalous couplings $\kappa_{g}^{t^{\prime}} / \Lambda=0.10 \mathrm{TeV}^{-1}, \kappa_{\gamma}^{t^{\prime}} / \Lambda=0.14 \mathrm{TeV}^{-1}, \kappa_{Z}^{t^{\prime}} / \Lambda=0.19 \mathrm{TeV}^{-1}$ and $\kappa_{g}^{b^{\prime}} / \Lambda=0.15 \mathrm{TeV}^{-1}, \kappa_{Z}^{b^{\prime}} / \Lambda=0.19 \mathrm{TeV}^{-1}, \kappa_{\gamma}^{b^{\prime}} / \Lambda=$ $0.30 \mathrm{TeV}^{-1}$ for the mass of $750 \mathrm{GeV}$ of the new heavy quarks $t^{\prime}$ and $b^{\prime}$ can be reached for an integrated luminosity of $L_{\mathrm{int}}=100 \mathrm{fb}$.
\end{abstract}

\section{Introduction}

The standard model (SM) of the strong and electroweak interactions describes successfully the phenomena of particle physics. However, there are many unanswered questions suggesting the SM to be an effective theory. In order to answer some of the problems with the SM, additional new fermions can be accommodated in many models beyond the SM (see [1-9] and references therein). The new heavy quarks could also be produced in pairs at the LHC with center of mass energy of $13 \mathrm{TeV}$. However, due to the expected smallness of the mixing between the new heavy quarks and known quarks, the decay modes can be quite different from the one relevant to charged weak interactions. A new symmetry beyond the $\mathrm{SM}$ is expected to explain the smallness of these mixings. The arguments given in [10] for anomalous interactions of the top quark are more valid for the new heavy quarks $t^{\prime}$ and $b^{\prime}$ due to their expected larger masses than the top quark.

The ATLAS experiment [11] and CMS experiment [12] have searched for the fourth generation of quarks and set limits on the mass of $m_{b^{\prime}}>480 \mathrm{GeV}$ and $m_{t^{\prime}}>570 \mathrm{GeV}$ at $\sqrt{s}=7 \mathrm{TeV}$. The pair production of new heavy quarks has been searched by the ATLAS experiment $[13,14]$ and the $m_{t^{\prime}}>656 \mathrm{GeV}$ mass limits are set at $\sqrt{s}=7 \mathrm{TeV}$. The CMS experiment has excluded $t^{\prime}$ masses below $557 \mathrm{GeV}$ [15]. The vector-like quarks have been searched by the ATLAS experiment $[16,17]$ and set bounds as $900 \mathrm{GeV}$ for charged current channel and $760 \mathrm{GeV}$ for neutral current channel at $\sqrt{s}=$ $7 \mathrm{TeV}$. The CMS experiment [18] has set the lower bounds on the mass of $685 \mathrm{GeV}$ at $\sqrt{s}=8 \mathrm{TeV}$. Some of the final states in the searches of new phenomena can also be considered in relation with the new heavy quarks.

The anomalous resonant productions of the fourth family quarks have been studied in $[19,20]$ at the LHC with $\sqrt{s}=$ $14 \mathrm{TeV}$. The possible single productions of fourth generation quarks via anomalous interactions at Tevatron have also been studied in [21]. The parameter space for the mixing of the fourth generation quarks has been presented in [22]. The $\mathrm{CP}$ violating flavor changing neutral current processes of the fourth generation quarks have been analyzed in [23], and the large mixing between fourth generation and first three generations has been excluded under the proposed fit conditions. Investigation of the parameter space favored by the precision electroweak data has been performed for the fourth SM family fermions in [24].

In this work, we present the analysis of anomalous productions and decay of new heavy quarks $t^{\prime}$ and $b^{\prime}$ at the LHC. We have performed the fast simulation for the signal and 
background. Any observations of the invariant mass peak in the range of 500-1000 GeV and excess in the events with the final states originating from $W b V$ and $b V$ can be interpreted as the signal for the new heavy quarks $t^{\prime}$ and $b^{\prime}$ via the anomalous interactions.

\section{New Heavy Quarks Anomalous Interactions}

A general theory that includes the standard model (SM) as its low energy limit can be written as an expansion series in powers of $\Lambda^{-1}$ with operators obeying the required symmetries. The dimension six gauge invariant operators can be built from the SM fields and they can induce dimension five operators after spontaneous symmetry breaking. The coefficients of the dimension five terms are related to those of dimension six operators, and they can lead to sizable effects in the heavy quark associated production in high energy collisions [25]. For our study, the effective Lagrangian with dimension five terms for the anomalous interactions among the new heavy quarks $\left(Q^{\prime} \equiv t^{\prime}\right.$ or $\left.b^{\prime}\right)$, ordinary quarks $q$, and the gauge bosons $V=\gamma, Z, g$ can be written explicitly:

$$
\begin{aligned}
L= & \sum_{q_{i}=u, c, t} \frac{\kappa_{\gamma}^{q_{i}}}{\Lambda} Q_{q_{i}} g_{e} \bar{t}^{\prime} \sigma_{\mu \nu} q_{i} F^{\mu \nu}+\sum_{q_{i}=u, c, t} \frac{\kappa_{z}^{q_{i}}}{2 \Lambda} g_{z} \bar{t}^{\prime} \sigma_{\mu \nu} q_{i} Z^{\mu \nu} \\
& +\sum_{q_{i}=u, c, t} \frac{\kappa_{g}^{q_{i}}}{2 \Lambda} g_{s} \bar{t}^{\prime} \sigma_{\mu \nu} \lambda_{a} q_{i} G_{a}^{\mu \nu}+\sum_{q_{i}=d, s, b} \frac{\kappa_{\gamma}^{q_{i}}}{\Lambda} Q_{q_{i}} g_{e} \bar{b}^{\prime} \sigma_{\mu \nu} q_{i} F^{\mu \nu} \\
& +\sum_{q_{i}=d, s, b} \frac{\kappa_{z}^{q_{i}}}{2 \Lambda} g_{z} \bar{b}^{\prime} \sigma_{\mu \nu} q_{i} Z^{\mu \nu}+\sum_{q_{i}=d, s, b} \frac{\kappa_{g}^{q_{i}}}{2 \Lambda} g_{s} \bar{b}^{\prime} \sigma_{\mu \nu} \lambda_{a} q_{i} G_{a}^{\mu \nu} \\
& + \text { h.c., }
\end{aligned}
$$

where $F^{\mu \nu}, Z^{\mu \nu}$, and $G^{\mu \nu}$ are the field strength tensors of the gauge bosons; $\sigma_{\mu \nu}=i\left(\gamma_{\mu} \gamma_{\nu}-\gamma_{\nu} \gamma_{\mu}\right) / 2 ; \lambda_{a}$ are the Gell-Mann matrices; $Q_{q}$ is the electric charge of the quark $(q) ; g_{e}, g_{Z}$, and $g_{s}$ are the electromagnetic, neutral weak, and strong coupling constants, respectively. $g_{Z}=g_{e} / \cos \theta_{w} \sin \theta_{w}$, where $\theta_{w}$ is the weak mixing angle. $\kappa_{\gamma}$ is the anomalous coupling with photon; $\kappa_{z}$ is for the $Z$ boson, and $\kappa_{g}$ is the coupling with gluon. Finally, $\Lambda$ is the cutoff scale for the new interactions.

\section{Decay Widths and Branchings}

For the decay channels $Q^{\prime} \rightarrow V q$ where $V \equiv \gamma, Z$, and $g$, we use the effective Lagrangian to calculate the anomalous decay widths:

$$
\begin{aligned}
& \Gamma\left(Q^{\prime} \longrightarrow g q\right)=\frac{2}{3}\left(\frac{\kappa_{g}^{q}}{\Lambda}\right)^{2} \alpha_{s} m_{Q^{\prime}}^{3} \lambda_{0}, \\
& \Gamma\left(Q^{\prime} \longrightarrow \gamma q\right)=\frac{1}{2}\left(\frac{\kappa_{\gamma}^{q}}{\Lambda}\right)^{2} \alpha_{e} Q_{q}^{2} m_{Q^{\prime}}^{3} \lambda_{0} \\
& \Gamma\left(Q^{\prime} \longrightarrow Z q\right)=\frac{1}{16}\left(\frac{\kappa_{Z}^{q}}{\Lambda}\right)^{2} \frac{\alpha_{e} m_{Q^{\prime}}^{3}}{\sin ^{2} \theta_{W} \cos ^{2} \theta_{W}} \lambda_{Z} \sqrt{\lambda_{r}}
\end{aligned}
$$

TABLE 1: Branching ratios (\%) and decay width of the new heavy quark $\left(t^{\prime}\right)$ with only anomalous interactions for PI parametrization and $\kappa / \Lambda=0.1 \mathrm{TeV}^{-1}$.

\begin{tabular}{lccccccc}
\hline $\begin{array}{l}\text { Mass } \\
(\mathrm{GeV})\end{array}$ & $g u(c)$ & $g t$ & $Z u(c)$ & $Z t$ & $\gamma u(c)$ & $\gamma t$ & $\begin{array}{c}\Gamma \\
(\mathrm{GeV})\end{array}$ \\
\hline 500 & 33.5 & 22.9 & 2.86 & 1.82 & 0.92 & 0.63 & 0.23 \\
600 & 32.3 & 25.0 & 2.86 & 2.13 & 0.91 & 0.70 & 0.41 \\
700 & 31.6 & 26.2 & 2.87 & 2.34 & 0.90 & 0.75 & 0.65 \\
800 & 31.1 & 27.0 & 2.89 & 2.48 & 0.90 & 0.78 & 0.97 \\
900 & 30.7 & 27.5 & 2.91 & 2.58 & 0.91 & 0.81 & 1.39 \\
1000 & 30.5 & 27.8 & 2.93 & 2.66 & 0.91 & 0.83 & 1.90 \\
\hline
\end{tabular}

with

$$
\begin{aligned}
& \lambda_{0}=1-\frac{3 m_{q}^{2}}{m_{\mathrm{Q}^{\prime}}^{2}}+\frac{3 m_{q}^{4}}{m_{\mathrm{Q}^{\prime}}^{4}}-\frac{m_{q}^{6}}{m_{\mathrm{Q}^{\prime}}^{6}}, \\
& \lambda_{r}=1+\frac{m_{Z}^{4}}{m_{\mathrm{Q}^{\prime}}^{4}}+\frac{m_{q}^{4}}{m_{\mathrm{Q}^{\prime}}^{4}}-\frac{2 m_{Z}^{2}}{m_{\mathrm{Q}^{\prime}}^{2}}-\frac{2 m_{q}^{2}}{m_{\mathrm{Q}^{\prime}}^{2}}-\frac{2 m_{Z^{2} m_{q}^{2}}^{2}}{m_{\mathrm{Q}^{\prime}}^{4}} \\
& \lambda_{Z}=2-\frac{m_{Z}^{2}}{m_{\mathrm{Q}^{\prime}}^{2}}-\frac{4 m_{q}^{2}}{m_{\mathrm{Q}^{\prime}}^{2}}+\frac{2 m_{q}^{4}}{m_{\mathrm{Q}^{\prime}}^{4}}-\frac{6 m_{q} m_{Z}^{2}}{m_{\mathrm{Q}^{\prime}}^{3}}-\frac{m_{Z^{2}}^{2} m_{t}^{2}}{m_{\mathrm{Q}^{\prime}}^{4}}-\frac{m_{Z}^{4}}{m_{\mathrm{Q}^{\prime}}^{4}}
\end{aligned}
$$

The anomalous decay widths in different channels are proportional to $\Lambda^{-2}$, and they are assumed to be dominant for $\kappa / \Lambda>0.1 \mathrm{TeV}^{-1}$ over the charged current channels. In this case, if we take all the anomalous coupling equal then the branching ratios will be nearly independent of $\kappa / \Lambda$. We have used three parametrization sets entitled PI, PII, and PIII. For the PI parametrization, we assume the constant value $\kappa_{i} / \Lambda=$ $0.1 \mathrm{TeV}^{-1}$, and PII has the parameters $\kappa_{i} / \Lambda=0.1 \lambda^{4-i} \mathrm{TeV}^{-1}$ with $\lambda=0.5$. For PIII we take the couplings $\kappa_{i} / \Lambda=$ $0.5 \lambda^{4-i} \mathrm{TeV}^{-1}$ with the same value of $\lambda$. The index $i$ is the generation number.

Tables 1 and 2 present the decay width and branching ratios of the new heavy quark $t^{\prime}$ through anomalous interactions for the parametrization PI, PII, and PIII, respectively. Taking the anomalous coupling $\kappa / \Lambda=0.1 \mathrm{TeV}^{-1}$ we calculate the $t^{\prime}$ decay width $\Gamma=0.65 \mathrm{GeV}$ and $1.90 \mathrm{GeV}$ for $m_{t^{\prime}}=$ $700 \mathrm{GeV}$ and $1000 \mathrm{GeV}$, respectively. The branching into $t^{\prime} \rightarrow$ $q g$ channel is the largest and branching into $t^{\prime} \rightarrow q \gamma$ channel is the smallest for equal anomalous couplings with the parametrization PI. On the other hand, PII and PIII parametrization give higher branching ratios into $t V(V=$ $g, Z, \gamma)$ than $q V(q=u, c)$ channels due to $\lambda^{4-i}$ factor in the parametrization.

For the new heavy quark $b^{\prime}$ the decay width and branching ratios are presented in Tables 3 and 4 for the parametrizations PI, PII, and PIII, respectively. We calculate the $b^{\prime}$ decay width, by taking the anomalous couplings $\kappa / \Lambda=0.1 \mathrm{TeV}^{-1}$, $\Gamma=0.68 \mathrm{GeV}$, and $1.92 \mathrm{GeV}$ for $m_{b^{\prime}}=700 \mathrm{GeV}$ and $1000 \mathrm{GeV}$, respectively. The branching for $b^{\prime} \rightarrow q g$ is the largest (30\%) and it is the smallest for $b^{\prime} \rightarrow q \gamma(0.2 \%)$ channel for equal anomalous couplings with the parametrization PI. For PII 
TABLE 2: The same as Table 1, but for PII (PIII) parametrization.

\begin{tabular}{lcccccccccc}
\hline Mass $(\mathrm{GeV})$ & $g u$ & $g c$ & $g t$ & $Z u$ & $Z c$ & $Z t$ & $\gamma u$ & $\gamma c$ & $\gamma t$ & $\Gamma(\mathrm{GeV})$ \\
\hline 500 & 5.66 & 22.60 & 61.90 & 0.48 & 1.93 & 4.92 & 0.15 & 0.62 & 1.71 & $0.021(0.558)$ \\
600 & 5.17 & 20.70 & 63.90 & 0.46 & 1.83 & 5.46 & 0.14 & 0.58 & 1.80 & $0.040(1.024)$ \\
700 & 4.90 & 19.60 & 64.90 & 0.44 & 1.78 & 5.79 & 0.14 & 0.56 & 1.87 & $0.066(1.68)$ \\
800 & 4.73 & 18.90 & 65.60 & 0.44 & 1.76 & 6.02 & 0.14 & 0.55 & 1.91 & $0.100(2.561)$ \\
900 & 4.61 & 18.40 & 65.90 & 0.44 & 1.74 & 6.19 & 0.13 & 0.54 & 1.95 & $0.145(3.680)$ \\
1000 & 4.53 & 18.10 & 66.20 & 0.43 & 1.74 & 6.32 & 0.13 & 0.54 & 1.98 & $0.200(5.070)$ \\
\hline
\end{tabular}

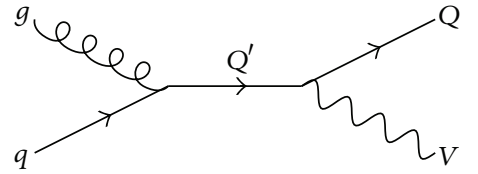

(a)

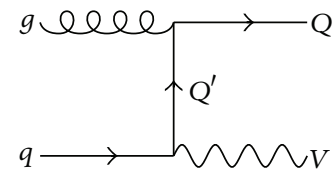

(b)

Figure 1: Diagrams for the subprocess $g q \rightarrow V Q$ with anomalous vertices $Q^{\prime} q V$ and $Q^{\prime} Q V$ (where $Q^{\prime}$ can be the new heavy quark $b^{\prime}$ or $t^{\prime}$ depending on the type of light $(q)$ or heavy $(Q \equiv t, b)$ quarks, resp.).

TABLE 3: Branching ratios (\%) and decay width of the new heavy quark $\left(b^{\prime}\right)$ with only anomalous interactions for PI parametrization and $\kappa / \Lambda=0.1 \mathrm{TeV}^{-1}$.

\begin{tabular}{lcccc}
\hline $\begin{array}{l}\text { Mass } \\
(\mathrm{GeV})\end{array}$ & $g d(s, b)$ & $Z d(s, b)$ & $\gamma d(s, b)$ & $\Gamma(\mathrm{GeV})$ \\
\hline 500 & 30.50 & 2.60 & 0.21 & 0.257 \\
600 & 30.40 & 2.69 & 0.21 & 0.436 \\
700 & 30.40 & 2.76 & 0.22 & 0.682 \\
800 & 30.30 & 2.82 & 0.22 & 1.005 \\
900 & 30.20 & 2.86 & 0.22 & 1.415 \\
1000 & 30.20 & 2.90 & 0.23 & 1.921 \\
\hline
\end{tabular}

and PIII parametrization the branching ratios into $b V(V=$ $g, Z, \gamma)$ are larger than $q V(q=d, s)$ channels. The $t^{\prime}$ and $b^{\prime}$ decay widths are about the same values for PII and PIII parametrization.

\section{The Cross Sections}

In order to study the new heavy quark productions at the LHC, we have used effective anomalous interaction vertices and implemented these vertices into the CalcHEP package [26]. In all of the numerical calculations, the parton distribution functions are set to the CTEQ6L parametrization [27]. The new heavy quarks can be produced through their anomalous couplings to the ordinary quarks and neutral vector bosons as shown in Figure 1.

Total cross sections for the productions of new heavy quarks $t^{\prime}$ and $b^{\prime}$ are given in Tables 5 and 6 for the parametrizations PI, PII, and PIII, at the center of mass energy of $8 \mathrm{TeV}$ and $13 \mathrm{TeV}$. For an illustration, taking the mass of new heavy quarks as $700 \mathrm{GeV}$ the cross section of $t^{\prime}\left(b^{\prime}\right)$ production is calculated as $8.50 \mathrm{pb}(10.03 \mathrm{pb})$ for the parametrization PIII at $\sqrt{s}=13 \mathrm{TeV}$. It can be seen from Tables 5 and 6 that the cross section decreases while the

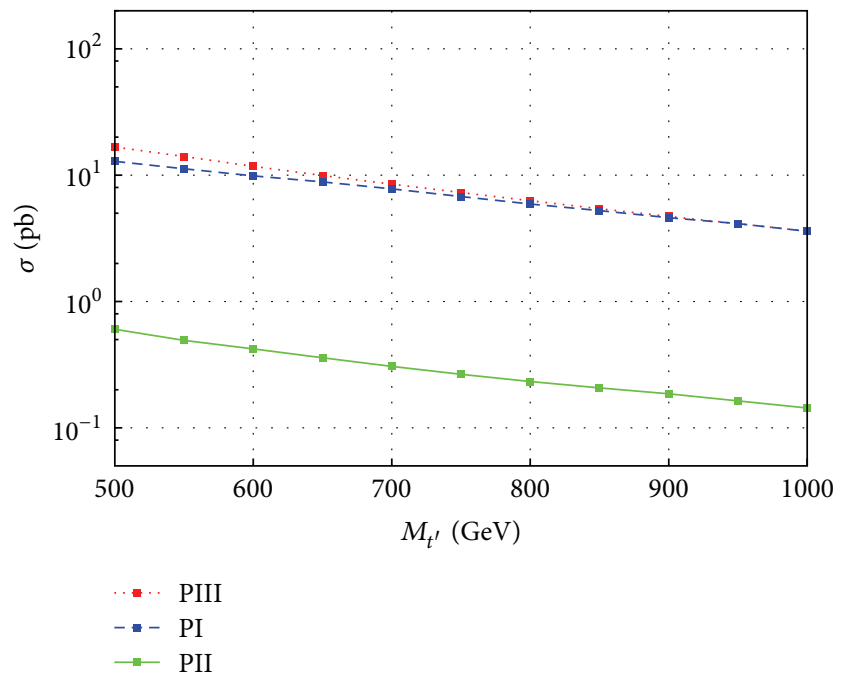

Figure 2: The cross section for the process $p p \rightarrow t V+X$ depending on the mass for parameter sets PI, PII, and PIII at the center of mass energy $\sqrt{s}=13 \mathrm{TeV}$.

mass of the new heavy quark increases. The cross section for $t^{\prime}$ production is larger than the $b^{\prime}$ production with a factor of 1.2-1.8 (0.7-1.0) for PI (PII and PIII) parametrization depending on the considered mass range at $\sqrt{s}=13 \mathrm{TeV}$. The general behaviour of the production cross sections depending on the mass of new heavy quarks is presented in Figures 2 and 3 for different parametrizations.

4.1. Analysis of the Process $p p \rightarrow W^{+} b V+X(V=g, Z, \gamma)$ for $t^{\prime}$ Signal. The signal process $p p \rightarrow W^{+} b V+X(V=g, Z, \gamma)$ includes the $t^{\prime}$ exchange in both the $s$-channel and $t$-channel. The $s$-channel contribution to the signal process would appear itself as resonance around the $t^{\prime}$ mass value in the $W b V$ invariant mass. The $t$-channel gives the nonresonant 
TABle 4: The same as Table 3, but for PII (PIII) parametrization.

\begin{tabular}{lcccccccccc}
\hline Mass $(\mathrm{GeV})$ & $g d$ & $g s$ & $g b$ & $Z d$ & $Z s$ & $Z b$ & $\gamma d$ & $\gamma s$ & $\gamma b$ & $\Gamma(\mathrm{GeV})$ \\
\hline 500 & 4.36 & 17.40 & 69.80 & 0.37 & 1.49 & 5.95 & 0.030 & 0.12 & 0.48 & $0.028(0.704)$ \\
600 & 4.35 & 17.40 & 69.50 & 0.38 & 1.54 & 6.16 & 0.030 & 0.12 & 0.49 & $0.047(1.194)$ \\
700 & 4.34 & 17.30 & 69.40 & 0.39 & 1.58 & 6.31 & 0.031 & 0.12 & 0.50 & $0.074(1.866)$ \\
800 & 4.33 & 17.30 & 69.20 & 0.40 & 1.61 & 6.44 & 0.031 & 0.12 & 0.50 & $0.110(2.749)$ \\
900 & 4.32 & 17.30 & 69.10 & 0.41 & 1.64 & 6.54 & 0.032 & 0.13 & 0.51 & $0.154(3.869)$ \\
1000 & 4.32 & 17.30 & 69.00 & 0.41 & 1.66 & 6.63 & 0.032 & 0.13 & 0.52 & $0.210(5.253)$ \\
\hline
\end{tabular}

TABLE 5: The cross sections (in pb) of new heavy quark $t^{\prime}$ production without cuts for PI, PII, and PIII parametrizations at the center of mass energy $13 \mathrm{TeV}(8 \mathrm{TeV})$, respectively.

\begin{tabular}{lccc}
\hline Mass $(\mathrm{GeV})$ & PI & PII & PIII \\
& $\sqrt{s}=13 \mathrm{TeV}(8 \mathrm{TeV})$ & $\sqrt{s}=13 \mathrm{TeV}(8 \mathrm{TeV})$ & $16.736(6.113)$ \\
500 & $13.733(5.30)$ & $0.664(0.244)$ & $11.770(4.031)$ \\
600 & $10.362(3.72)$ & $0.464(0.159)$ & $8.502(2.718)$ \\
700 & $7.825(2.64)$ & $0.337(0.109)$ & $6.276(1.882)$ \\
800 & $5.961(1.89)$ & $0.250(0.075)$ & $4.701(1.326)$ \\
900 & $4.602(1.36)$ & $0.189(0.053)$ & $3.609(0.950)$ \\
1000 & $3.593(0.98)$ & $0.144(0.038)$ & $\mathrm{TeV}(8 \mathrm{TeV})$ \\
\hline
\end{tabular}

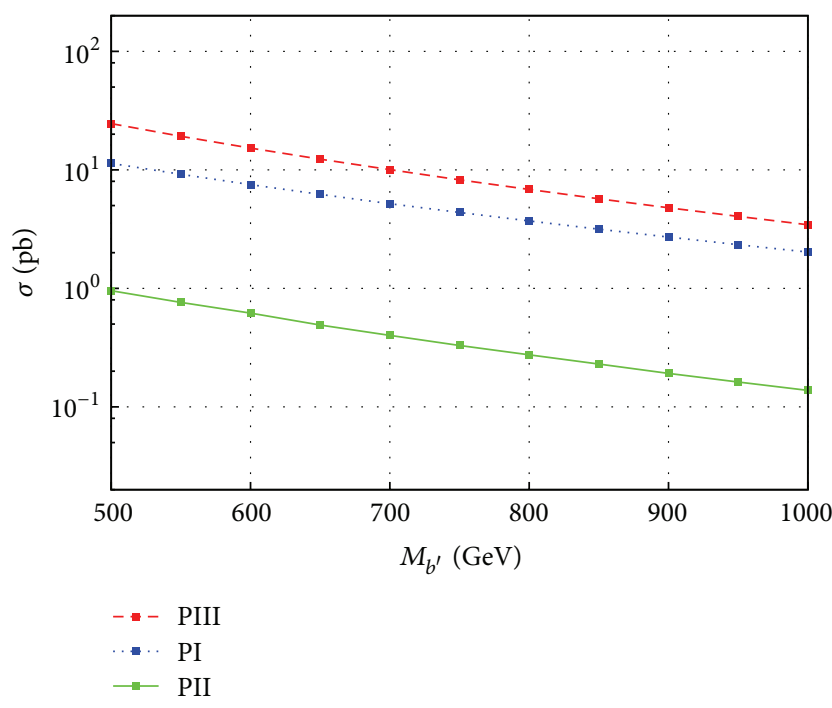

FIGURE 3: The cross section for the process $p p \rightarrow b V+X$ depending on the new heavy quark mass for parameter sets PI, PII, and PII at the center of mass energy $\sqrt{s}=13 \mathrm{TeV}$.

contribution. We consider that the $W$ boson decays into lepton + missing transverse momentum with the branching ratio of $21 \%$ and $Z$ boson decays into dilepton with the branching ratio of $6.7 \%$. In our analyses, we consider the $t^{\prime}$ signal in the $l+b_{\text {jet }}+\gamma+$ MET, $l+b_{\text {jet }}+j+$ MET, and $3 l+b_{\text {jet }}+$ MET channels, where $l=e, \mu$. However, if one takes the hadronic $W$ decay, the signal will be enhanced by a factor of $\mathrm{BR}(W \rightarrow$ hadrons $) / \mathrm{BR}(W \rightarrow l v)$.

We have obtained the cross sections by using the cuts pseudorapidity $\left|\eta_{j, \gamma}\right|<2.5$ and transverse momentum $p_{T}^{j, \gamma}>$ 20-200 GeV for jets and photon, in Table 7 (Tables 8 and 9)

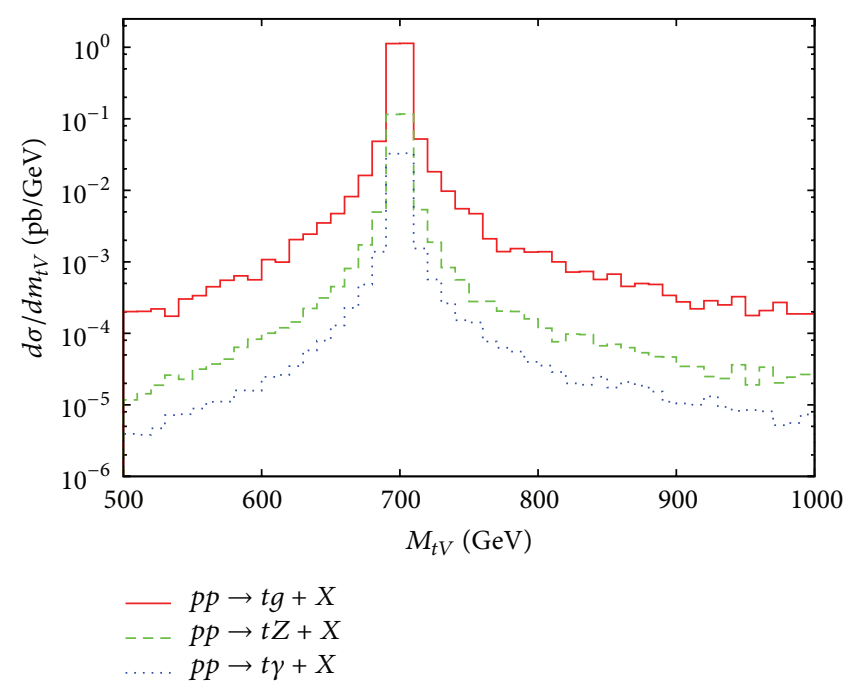

FIGURE 4: Invariant mass distributions $m_{t V}$ (where $V=\gamma, g$, and $Z$ ) for PI parametrization of the signal with $\kappa / \Lambda=0.2 \mathrm{TeV}^{-1}$ and $m_{t^{\prime}}=700 \mathrm{GeV}$ at the center of mass energy $\sqrt{s}=13 \mathrm{TeV}$.

for PI (PII, PIII) parametrization, respectively. Invariant mass distribution of the $t V$ (where $V=\gamma, g$, and $Z$ ) system is shown in Figure 4 for PI parametrization of the signal with $\kappa / \Lambda=0.2 \mathrm{TeV}^{-1}$ and $m_{t^{\prime}}=700 \mathrm{GeV}$ at the center of mass energy $\sqrt{s}=13 \mathrm{TeV}$. It appears from signal significance calculations that the optimized transverse momentum cut is $p_{T}^{j, \gamma}>100 \mathrm{GeV}$ for $t^{\prime}$ analyses.

The backgrounds for the final state $W^{+} b(\bar{b}) V$ (where $V \equiv$ photon, jet, and $Z$ boson) are given in Table 10 . We apply the following cuts to the final state photon and jets as $\left|\eta_{j, \gamma}\right|<2.5$ and $p_{T}^{j, \gamma}>20-200 \mathrm{GeV}$. For the background cross section 
TABLE 6: The cross sections (in $\mathrm{pb}$ ) of new heavy quark $b^{\prime}$ production without cuts for PI, PII, and PIII parametrizations at the center of mass energy of $13 \mathrm{TeV}(8 \mathrm{TeV})$, respectively.

\begin{tabular}{lccc}
\hline Mass $(\mathrm{GeV})$ & PI & PII & PIII \\
& $\sqrt{s}=13 \mathrm{TeV}(8 \mathrm{TeV})$ & $\sqrt{s}=13 \mathrm{TeV}(8 \mathrm{TeV})$ & $24.474(7.114)$ \\
500 & $11.340(3.913)$ & $0.970(0.285)$ & $13.290(4.09)$ \\
600 & $7.495(2.410)$ & $0.607(0.162)$ & $10.031(2.483)$ \\
700 & $5.179(1.546)$ & $0.412(0.099)$ & $6.832(1.566)$ \\
800 & $3.697(1.025)$ & $0.286(0.062)$ & $4.791(1.018)$ \\
900 & $2.707(0.697)$ & $0.1905(0.040)$ & $3.441(0.678)$ \\
1000 & $2.021(0.482)$ & $0.137(0.027)$ & $\mathrm{TeV})$ \\
\hline
\end{tabular}

TABLE 7: The cross sections (in pb) for $t^{\prime}$ signal in different decay channels for PI parametrization with $p_{T}$ cuts on the jets and photon and $\left|\eta_{j, \gamma}\right|<2.5$ at the center of mass energy $\sqrt{s}=13 \mathrm{TeV}$.

\begin{tabular}{|c|c|c|c|c|}
\hline \multirow{2}{*}{$\begin{array}{l}\text { Signal } \\
\text { Mass }(\mathrm{GeV})\end{array}$} & \multicolumn{4}{|c|}{ PI } \\
\hline & $p_{T}>20 \mathrm{GeV}$ & $p_{T}>50 \mathrm{GeV}$ & $p_{T}>100 \mathrm{GeV}$ & $p_{T}>200 \mathrm{GeV}$ \\
\hline \multicolumn{5}{|c|}{$p p \rightarrow W^{+} b \gamma+X$} \\
\hline 500 & $2.89 \times 10^{-1}$ & $2.10 \times 10^{-1}$ & $1.24 \times 10^{-1}$ & $1.02 \times 10^{-4}$ \\
\hline 600 & $2.43 \times 10^{-1}$ & $1.64 \times 10^{-1}$ & $1.19 \times 10^{-1}$ & $1.23 \times 10^{-2}$ \\
\hline 700 & $1.68 \times 10^{-1}$ & $1.2 \times 10^{-1}$ & $1.12 \times 10^{-1}$ & $2.25 \times 10^{-2}$ \\
\hline 800 & $1.30 \times 10^{-1}$ & $1.03 \times 10^{-1}$ & $7.53 \times 10^{-2}$ & $3.25 \times 10^{-2}$ \\
\hline 900 & $1.02 \times 10^{-1}$ & $8.08 \times 10^{-2}$ & $6.96 \times 10^{-2}$ & $3.02 \times 10^{-2}$ \\
\hline 1000 & $7.61 \times 10^{-2}$ & $6.35 \times 10^{-2}$ & $5.07 \times 10^{-2}$ & $2.94 \times 10^{-2}$ \\
\hline \multicolumn{5}{|c|}{$p p \rightarrow W^{+} b g+X$} \\
\hline 500 & $7.78 \times 10^{0}$ & $6.02 \times 10^{0}$ & $3.63 \times 10^{0}$ & $4.74 \times 10^{-3}$ \\
\hline 600 & $6.30 \times 10^{0}$ & $5.18 \times 10^{0}$ & $3.13 \times 10^{0}$ & $2.58 \times 10^{-1}$ \\
\hline 700 & $4.99 \times 10^{0}$ & $3.63 \times 10^{0}$ & $3.04 \times 10^{0}$ & $9.32 \times 10^{-1}$ \\
\hline 800 & $4.01 \times 10^{0}$ & $3.45 \times 10^{0}$ & $2.76 \times 10^{0}$ & $9.91 \times 10^{-1}$ \\
\hline 900 & $3.32 \times 10^{0}$ & $2.77 \times 10^{0}$ & $2.13 \times 10^{0}$ & $1.08 \times 10^{0}$ \\
\hline 1000 & $2.58 \times 10^{0}$ & $2.27 \times 10^{0}$ & $1.88 \times 10^{0}$ & $1.01 \times 10^{0}$ \\
\hline \multicolumn{5}{|c|}{$p p \rightarrow W^{+} b Z+X$} \\
\hline 500 & $7.96 \times 10^{-1}$ & $6.01 \times 10^{-1}$ & $3.01 \times 10^{-1}$ & $1.01 \times 10^{-4}$ \\
\hline 600 & $4.79 \times 10^{-1}$ & $3.86 \times 10^{-1}$ & $2.45 \times 10^{-1}$ & $2.71 \times 10^{-3}$ \\
\hline 700 & $3.99 \times 10^{-1}$ & $3.12 \times 10^{-1}$ & $2.39 \times 10^{-1}$ & $6.96 \times 10^{-2}$ \\
\hline 800 & $3.31 \times 10^{-1}$ & $2.89 \times 10^{-1}$ & $2.09 \times 10^{-1}$ & $8.05 \times 10^{-2}$ \\
\hline 900 & $2.73 \times 10^{-1}$ & $2.73 \times 10^{-1}$ & $1.91 \times 10^{-1}$ & $9.54 \times 10^{-2}$ \\
\hline 1000 & $2.23 \times 10^{-1}$ & $2.02 \times 10^{-1}$ & $1.61 \times 10^{-1}$ & $9.10 \times 10^{-2}$ \\
\hline
\end{tabular}

estimates, we assume the efficiency for $b$-tagging to be $\varepsilon_{b}=$ $50 \%$ and the rejection ratios to be $10 \%$ for $c(\bar{c})$ quark jets and $1 \%$ for light quark jets since they are assumed to be mistagged as $b$-jets.

In order to find the discovery limits we use the statistical significance [28] defined as

$$
S S=\sqrt{2\left[(S+B) \ln \left(1+\frac{S}{B}\right)-S\right]},
$$

where $S$ and $B$ are the numbers of the signal and background events, respectively. In Figures 5-7, the integrated luminosity required to reach $3 \sigma$ significance for the signal of $t^{\prime}$ anomalous interactions is shown for parametrizations PI, PII, and PIII at the LHC with $\sqrt{s}=13 \mathrm{TeV}$. It is seen from these figures that the channel $t^{\prime} \rightarrow t Z$ requires more integrated luminosity than the other channels. By requiring the signal significance $S S=3$, the contour plots of $\kappa / \Lambda$ and mass of $t^{\prime}$ quark are presented in Figure 8. The results show that one can discover the $t^{\prime}$ quark anomalous couplings $\kappa / \Lambda$ down to $0.1 \mathrm{TeV}^{-1}$ in the $\mathrm{tg}$ channel for $m_{t^{\prime}}=750 \mathrm{GeV}$.

4.1.1. Simulation for $t^{\prime}$ Signal. In order to include detector effects in the simulation, we have generated $t V$ (where $V=$ $\gamma, g$, and $Z$ ) signal events for each subprocess and they are mixed using the "event_mixer" script which can be found within the CALCHEP package [26]. For further decay and hadronization these events are passed to PYTHIA [29] and simulated with the PGS4 program [30] using generic LHC 
TABLE 8: The same as Table 7, but for parametrization PII.

\begin{tabular}{|c|c|c|c|c|}
\hline \multirow{2}{*}{$\begin{array}{l}\text { Signal } \\
\text { Mass }(\mathrm{GeV})\end{array}$} & \multicolumn{4}{|c|}{ PII } \\
\hline & $p_{T}>20 \mathrm{GeV}$ & $p_{T}>50 \mathrm{GeV}$ & $p_{T}>100 \mathrm{GeV}$ & $p_{T}>200 \mathrm{GeV}$ \\
\hline \multicolumn{5}{|c|}{$p p \rightarrow W^{+} b \gamma+X$} \\
\hline 500 & $6.78 \times 10^{-3}$ & $5.07 \times 10^{-3}$ & $3.45 \times 10^{-3}$ & $2.64 \times 10^{-7}$ \\
\hline 600 & $6.57 \times 10^{-3}$ & $5.42 \times 10^{-3}$ & $3.47 \times 10^{-3}$ & $5.34 \times 10^{-4}$ \\
\hline 700 & $5.02 \times 10^{-3}$ & $4.31 \times 10^{-3}$ & $3.04 \times 10^{-3}$ & $8.73 \times 10^{-4}$ \\
\hline 800 & $3.91 \times 10^{-3}$ & $3.76 \times 10^{-3}$ & $2.56 \times 10^{-3}$ & $1.03 \times 10^{-3}$ \\
\hline 900 & $3.03 \times 10^{-3}$ & $2.68 \times 10^{-3}$ & $2.11 \times 10^{-3}$ & $1.01 \times 10^{-3}$ \\
\hline 1000 & $2.40 \times 10^{-3}$ & $2.43 \times 10^{-3}$ & $1.77 \times 10^{-3}$ & $9.98 \times 10^{-4}$ \\
\hline \multicolumn{5}{|c|}{$p p \rightarrow W^{+} b g+X$} \\
\hline 500 & $3.47 \times 10^{-1}$ & $2.68 \times 10^{-1}$ & $1.52 \times 10^{-1}$ & $5.30 \times 10^{-6}$ \\
\hline 600 & $2.51 \times 10^{-1}$ & $2.12 \times 10^{-1}$ & $1.35 \times 10^{-1}$ & $2.01 \times 10^{-2}$ \\
\hline 700 & $1.87 \times 10^{-1}$ & $1.6 \times 10^{-1}$ & $1.16 \times 10^{-1}$ & $3.42 \times 10^{-2}$ \\
\hline 800 & $1.46 \times 10^{-1}$ & $1.25 \times 10^{-1}$ & $9.39 \times 10^{-2}$ & $4.03 \times 10^{-2}$ \\
\hline 900 & $1.12 \times 10^{-1}$ & $1.08 \times 10^{-1}$ & $7.80 \times 10^{-2}$ & $3.86 \times 10^{-2}$ \\
\hline 1000 & $9.35 \times 10^{-2}$ & $8.37 \times 10^{-2}$ & $6.62 \times 10^{-2}$ & $3.68 \times 10^{-2}$ \\
\hline \multicolumn{5}{|c|}{$p p \rightarrow W^{+} b Z+X$} \\
\hline 500 & $2.10 \times 10^{-2}$ & $1.77 \times 10^{-2}$ & $1.16 \times 10^{-2}$ & $2.64 \times 10^{-7}$ \\
\hline 600 & $1.95 \times 10^{-2}$ & $1.75 \times 10^{-2}$ & $1.14 \times 10^{-2}$ & $1.34 \times 10^{-3}$ \\
\hline 700 & $1.73 \times 10^{-2}$ & $1.43 \times 10^{-2}$ & $1.00 \times 10^{-2}$ & $2.9 \times 10^{-3}$ \\
\hline 800 & $1.34 \times 10^{-2}$ & $1.19 \times 10^{-2}$ & $8.89 \times 10^{-3}$ & $3.42 \times 10^{-3}$ \\
\hline 900 & $1.06 \times 10^{-2}$ & $9.55 \times 10^{-3}$ & $7.63 \times 10^{-3}$ & $3.37 \times 10^{-3}$ \\
\hline 1000 & $8.09 \times 10^{-3}$ & $7.58 \times 10^{-3}$ & $6.31 \times 10^{-3}$ & $3.23 \times 10^{-3}$ \\
\hline
\end{tabular}

TABle 9: The same as Table 7, but for parametrization PIII.

\begin{tabular}{|c|c|c|c|c|}
\hline \multirow{2}{*}{$\begin{array}{l}\text { Signal } \\
\text { Mass }(\mathrm{GeV})\end{array}$} & \multicolumn{4}{|c|}{ PIII } \\
\hline & $p_{T}>20 \mathrm{GeV}$ & $p_{T}>50 \mathrm{GeV}$ & $p_{T}>100 \mathrm{GeV}$ & $p_{T}>200 \mathrm{GeV}$ \\
\hline \multicolumn{5}{|c|}{$p p \rightarrow W^{+} b \gamma+X$} \\
\hline 500 & $2.60 \times 10^{-1}$ & $2.78 \times 10^{-1}$ & $1.08 \times 10^{-1}$ & $1.59 \times 10^{-4}$ \\
\hline 600 & $1.78 \times 10^{-1}$ & $1.61 \times 10^{-1}$ & $1.01 \times 10^{-1}$ & $1.42 \times 10^{-2}$ \\
\hline 700 & $1.56 \times 10^{-1}$ & $1.35 \times 10^{-1}$ & $9.33 \times 10^{-2}$ & $2.72 \times 10^{-2}$ \\
\hline 800 & $1.17 \times 10^{-1}$ & $1.06 \times 10^{-1}$ & $7.84 \times 10^{-2}$ & $3.32 \times 10^{-2}$ \\
\hline 900 & $9.04 \times 10^{-2}$ & $8.42 \times 10^{-2}$ & $6.68 \times 10^{-2}$ & $3.25 \times 10^{-2}$ \\
\hline 1000 & $7.60 \times 10^{-2}$ & $6.76 \times 10^{-2}$ & $5.16 \times 10^{-2}$ & $3.17 \times 10^{-2}$ \\
\hline \multicolumn{5}{|c|}{$p p \rightarrow W^{+} b g+X$} \\
\hline 500 & $8.39 \times 10^{0}$ & $6.49 \times 10^{0}$ & $3.86 \times 10^{0}$ & $4.65 \times 10^{-3}$ \\
\hline 600 & $6.10 \times 10^{0}$ & $5.78 \times 10^{0}$ & $3.81 \times 10^{0}$ & $5.56 \times 10^{-1}$ \\
\hline 700 & $5.39 \times 10^{0}$ & $4.64 \times 10^{0}$ & $3.41 \times 10^{0}$ & $9.70 \times 10^{-1}$ \\
\hline 800 & $3.94 \times 10^{0}$ & $3.54 \times 10^{0}$ & $2.73 \times 10^{0}$ & $1.05 \times 10^{0}$ \\
\hline 900 & $3.24 \times 10^{0}$ & $2.76 \times 10^{0}$ & $2.27 \times 10^{0}$ & $1.07 \times 10^{0}$ \\
\hline 1000 & $2.33 \times 10^{0}$ & $2.29 \times 10^{0}$ & $1.84 \times 10^{0}$ & $9.98 \times 10^{-1}$ \\
\hline \multicolumn{5}{|c|}{$p p \rightarrow W^{+} b Z+X$} \\
\hline 500 & $7.72 \times 10^{-1}$ & $1.01 \times 10^{0}$ & $2.17 \times 10^{-1}$ & $6.27 \times 10^{-4}$ \\
\hline 600 & $6.24 \times 10^{-1}$ & $3.85 \times 10^{-1}$ & $2.92 \times 10^{-1}$ & $3.20 \times 10^{-2}$ \\
\hline 700 & $5.00 \times 10^{-1}$ & $3.05 \times 10^{-1}$ & $2.86 \times 10^{-1}$ & $5.80 \times 10^{-2}$ \\
\hline 800 & $3.78 \times 10^{-1}$ & $2.50 \times 10^{-1}$ & $2.42 \times 10^{-1}$ & $9.64 \times 10^{-2}$ \\
\hline 900 & $3.04 \times 10^{-1}$ & $1.67 \times 10^{-1}$ & $2.06 \times 10^{-1}$ & $9.62 \times 10^{-2}$ \\
\hline 1000 & $2.51 \times 10^{-1}$ & $1.29 \times 10^{-1}$ & $1.48 \times 10^{-1}$ & $9.61 \times 10^{-2}$ \\
\hline
\end{tabular}


TABLE 10: The cross sections (in pb) for the relevant backgrounds $\left(W^{+} b(\bar{b}) V, W^{+} c(\bar{c}) V\right.$, and $W^{+} j V$, where $V=$ photon, jet, and $Z$ boson) with $p_{T}$ cuts on the jets at the center of mass energy $\sqrt{s}=13 \mathrm{TeV}$.

\begin{tabular}{lcccc}
\hline Background & $p_{T}>20 \mathrm{GeV}$ & $p_{T}>50 \mathrm{GeV}$ & $p_{T}>100 \mathrm{GeV}$ & $p_{T}>200 \mathrm{GeV}$ \\
\hline$p p \rightarrow W^{+} b \gamma+X$ & $2.37 \times 10^{-3}$ & $3.62 \times 10^{-4}$ & $6.17 \times 10^{-5}$ & $6.25 \times 10^{-2}$ \\
$p p \rightarrow W^{+} \bar{c} \gamma+X$ & $4.15 \times 10^{0}$ & $4.59 \times 10^{-1}$ & $7.33 \times 10^{-1}$ & $6.21 \times 10^{-3}$ \\
$p p \rightarrow W^{+} j \gamma+X$ & $2.63 \times 10^{1}$ & $4.30 \times 10^{0}$ & $6.11 \times 10^{0}$ & $1.27 \times 10^{-1}$ \\
$p p \rightarrow W^{+} b(\bar{b}) j+X$ & $7.26 \times 10^{1}$ & $3.02 \times 10^{1}$ & $1.79 \times 10^{1}$ & $9.74 \times 10^{-1}$ \\
$p p \rightarrow W^{+} c(\bar{c}) j+X$ & $5.98 \times 10^{2}$ & $9.65 \times 10^{1}$ & $1.61 \times 10^{2}$ & $2.42 \times 10^{0}$ \\
$p p \rightarrow W^{+} j j+X$ & $7.31 \times 10^{3}$ & $7.78 \times 10^{2}$ & $1.93 \times 10^{-4}$ & $2.58 \times 10^{1}$ \\
$p p \rightarrow W^{+} b Z+X$ & $6.26 \times 10^{-4}$ & $3.99 \times 10^{-4}$ & $1.66 \times 10^{-1}$ & $4.71 \times 10^{-5}$ \\
$p p \rightarrow W^{+} \bar{c} Z+X$ & $5.29 \times 10^{-1}$ & $3.40 \times 10^{-1}$ & $2.49 \times 10^{0}$ & $4.15 \times 10^{-2}$ \\
$p p \rightarrow W^{+} j Z+X$ & $8.59 \times 10^{0}$ & $4.83 \times 10^{0}$ & $7.91 \times 10^{-1}$ \\
\hline
\end{tabular}

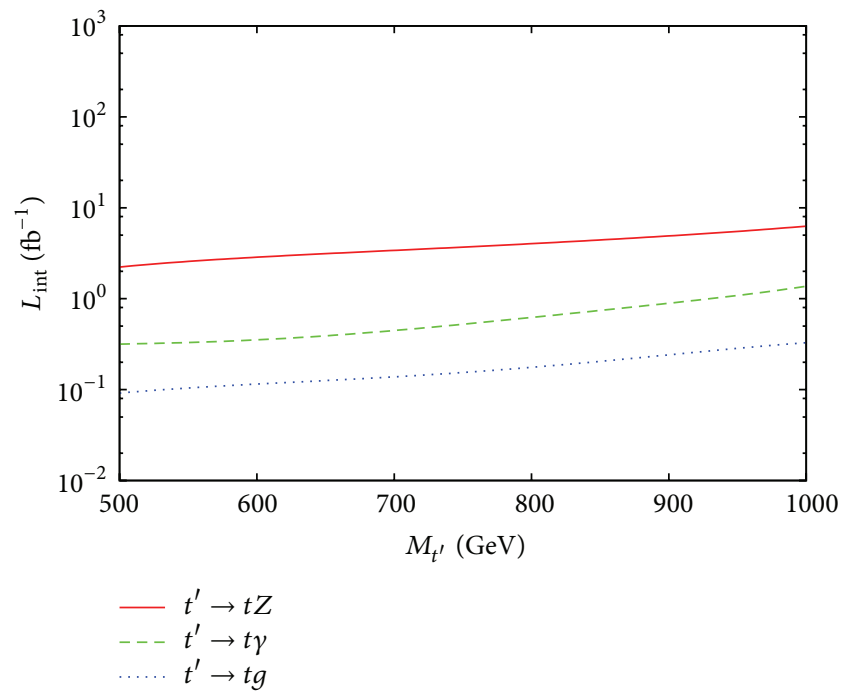

FIGURE 5: Integrated luminosity required to reach $3 \sigma$ significance for the signal of $t^{\prime}$ anomalous interactions for parametrization PI at the LHC with $\sqrt{s}=13 \mathrm{TeV}$.

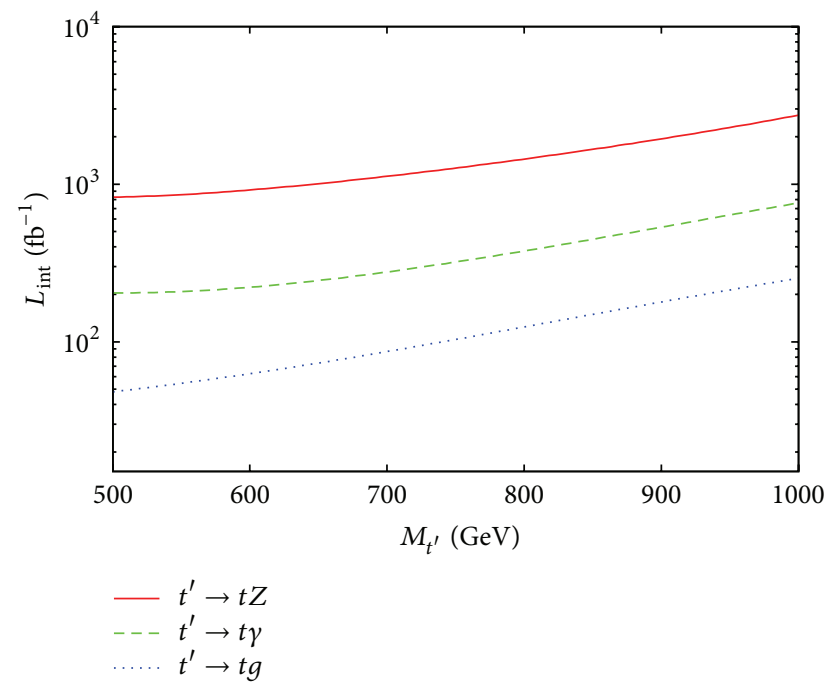

Figure 6: The same as Figure 5, but for parametrization PII.

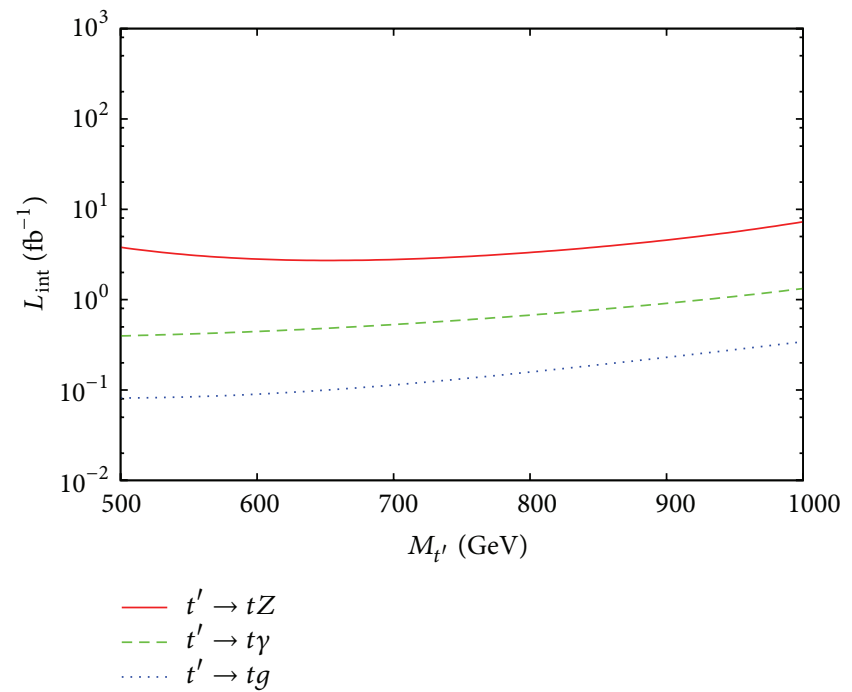

FIGURE 7: The same as Figure 5, but for parametrization PIII.

detector parameters. This fast simulation includes the important detector effects such as tracking, smearing effects of the calorimeters, resolution, and tag efficiencies. The EXROOTANALYSIS package [31] is used for the simulated events and the output is analyzed and histogrammed with the ROOT [32] macros. We consider jets (up to five), leptons (electrons or muons), photons, and missing transverse momentum within the simulated events for the $t \gamma, t j$, and $t Z$ events generation. The typical kinematical distributions are shown in Figures 9-10.

In the analysis, the signal (with $\kappa / \Lambda=0.2 \mathrm{TeV}^{-1}$ and $\left.m_{t^{\prime}}=700 \mathrm{GeV}\right)$ and the corresponding background $(\mathrm{WjV})$ are taken into account. The $s$-channel contribution to the signal process appears as a resonance around the $t^{\prime}$ mass value in the reconstructed invariant mass $m_{t^{\prime}}^{\text {rec }}$. The reconstructed mass distribution for the $t^{\prime}$ signal (reconstructed from a top quark and a vector boson) is shown in Figure 11.

Similar to the single top processes, the top quark in the final state is reconstructed from a leading jet (commonly $b_{\text {jet }}$ ) and a $W$ boson (which can be reconstructed from its leptonic or hadronic decay). For the $t \gamma$ production we require 


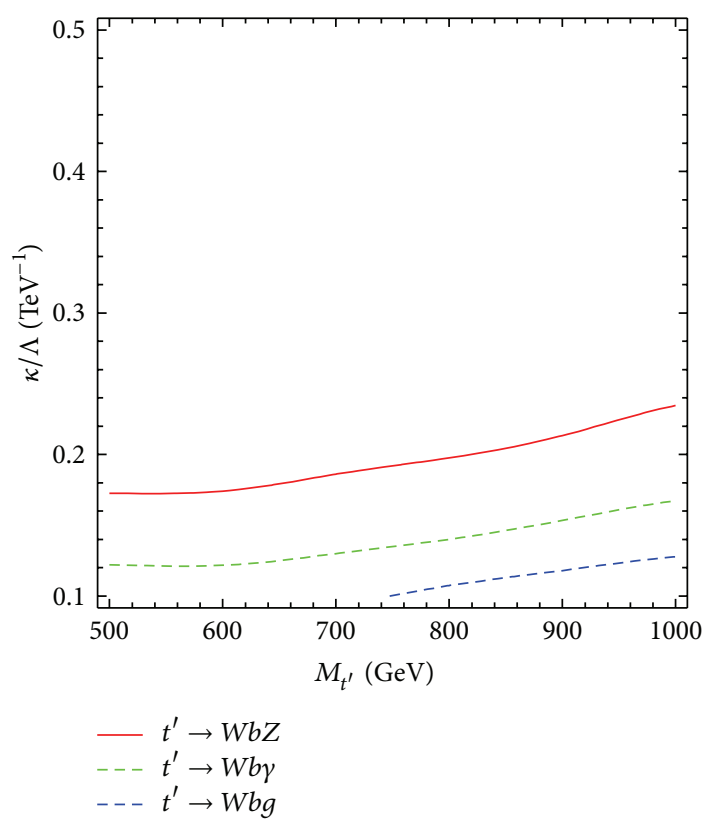

Figure 8: The contour plot of anomalous coupling and mass of new heavy quark $t^{\prime}$ for the dynamical parametrization explained in the text with a significance of $3 \sigma$ at $\sqrt{s}=13 \mathrm{TeV}$ and $L_{\text {int }}=100 \mathrm{fb}^{-1}$.

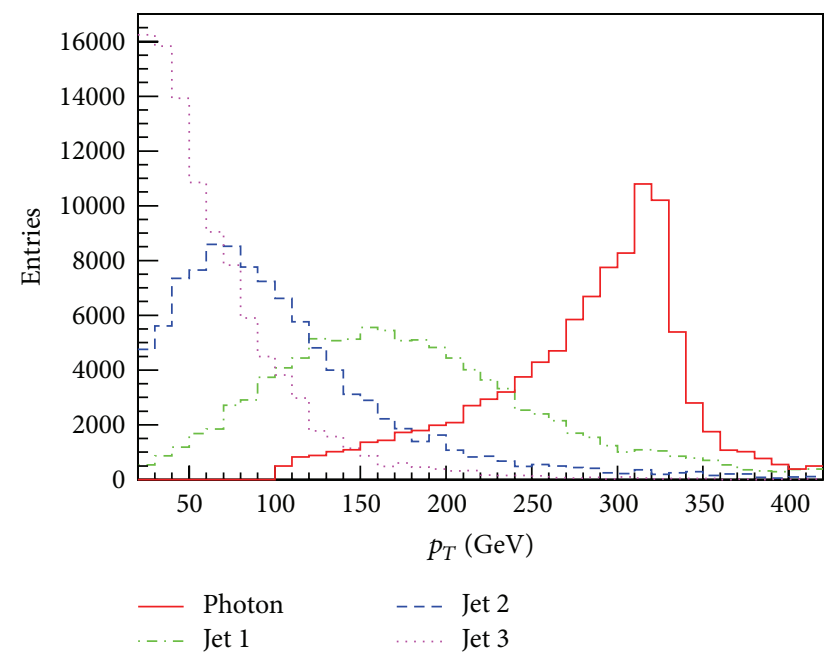

FIGURE 9: Transverse momentum distributions of leading jet (Jet 1) and other jets (Jet 2 and Jet 3 ) and photon for signal ( $t \gamma$ production) after detector simulation.

systematically the large transverse momentum of photon $\left(p_{T}^{\gamma}>100 \mathrm{GeV}\right)$, minimum jet transverse momentum $\left(p_{T}^{j}>\right.$ $20 \mathrm{GeV})$, and the pseudorapidity range $\left(\left|\eta^{\mathrm{j}, \gamma}\right|<2.5\right)$ in addition to the requirements on mass reconstruction of $W$ boson and top quark. The large $p_{T}^{\gamma}$ and the requirement of single $b$-tagging allow a better separation of the signal (for $t \gamma$ channel) from the background. Other channels for $t g$ and $t Z$ productions are more challenging due to a large number of jets, which require additional discriminators such as angular and/or total transverse energy variables. However, in order to get rid of the backgrounds from $W t$ and $t \bar{t}$ production

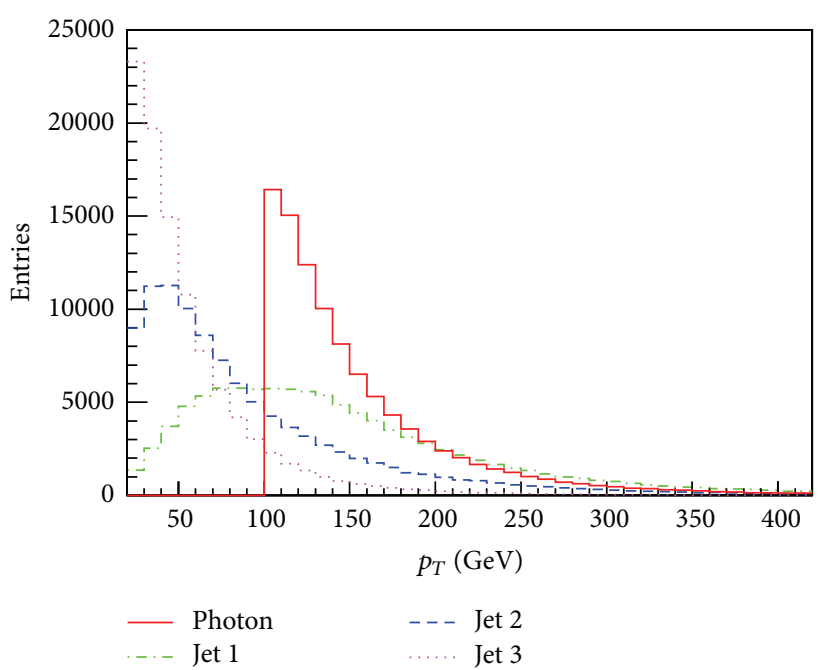

FIGURE 10: Transverse momentum distributions of leading jet (Jet 1) and other jets (Jet 2 and Jet 3 ) and photon for background (Wjy production).

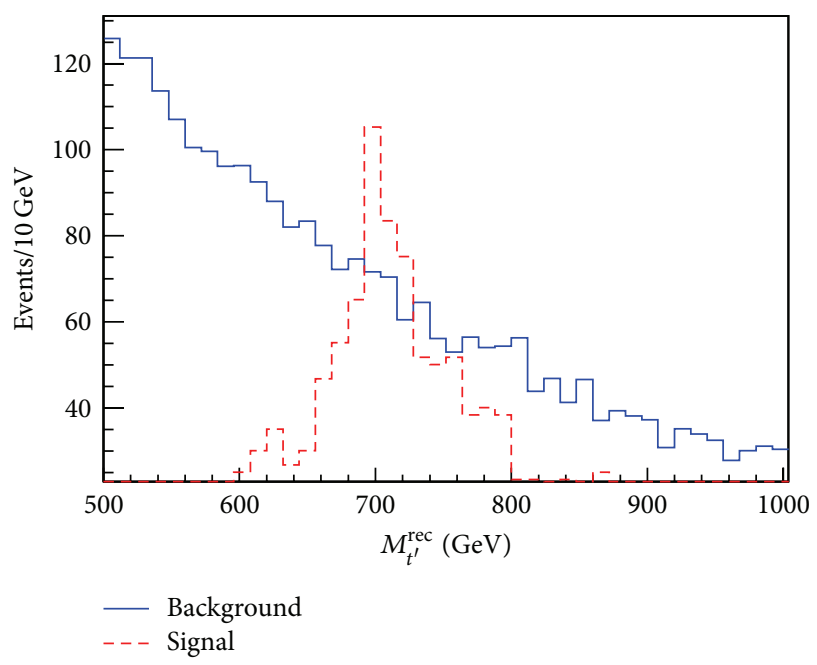

FIGURE 11: The reconstructed mass distributions for background and signal $(t \gamma)$ with $m_{t^{\prime}}=700 \mathrm{GeV}$ and $\kappa / \Lambda=0.15 \mathrm{TeV}^{-1}$.

(for a similar framework the production cross sections are about $25 \mathrm{pb}$ and $340 \mathrm{pb}$, resp.), one can consider the channel $3 l+b_{\text {jet }}+$ MET for a distinctive signal from the $t^{\prime} \rightarrow t Z$. An analysis of the investigation of single top production with similar backgrounds at the LHC can be found in [33-35].

4.2. Analysis of the Process $p p \rightarrow b V+X(V=g, Z, \gamma)$ for $b^{\prime}$ Signal. The signal process $p p \rightarrow b V+X(V=g, Z, \gamma)$ includes the new heavy quark $b^{\prime}$ exchange in both the $s$ channel and the $t$-channel. The $s$-channel contributes to the signal process as resonance around the $b^{\prime}$ mass value in the $b V$ invariant mass, while the $t$-channel contributes to the nonresonant behaviour. For this process, we consider the leptonic decay of $Z$ boson. In the analyses, we consider the $b /$ signal to be $b_{\text {jet }}+\gamma, b_{\text {jet }}+j$, and $b_{\text {jet }}+$ dilepton. 
TABLE 11: The cross sections (in pb) for $b^{\prime}$ signal in different decay channel for parametrization PI with $p_{T}$ cuts on the jets and photon and $\left|\eta_{j, \gamma}\right|<2.5$ at the center of mass energy $\sqrt{s}=13 \mathrm{TeV}$.

\begin{tabular}{|c|c|c|c|c|}
\hline \multirow{2}{*}{$\begin{array}{l}\text { Signal } \\
\text { Mass }(\mathrm{GeV})\end{array}$} & \multicolumn{4}{|c|}{ PI } \\
\hline & $p_{T}>20 \mathrm{GeV}$ & $p_{T}>50 \mathrm{GeV}$ & $p_{T}>100 \mathrm{GeV}$ & $p_{T}>200 \mathrm{GeV}$ \\
\hline \multicolumn{5}{|c|}{$p p \rightarrow b \gamma+X$} \\
\hline 500 & $5.64 \times 10^{-2}$ & $5.62 \times 10^{-2}$ & $5.49 \times 10^{-2}$ & $3.95 \times 10^{-2}$ \\
\hline 600 & $3.96 \times 10^{-2}$ & $3.96 \times 10^{-2}$ & $3.90 \times 10^{-2}$ & $3.33 \times 10^{-2}$ \\
\hline 700 & $2.87 \times 10^{-2}$ & $2.87 \times 10^{-2}$ & $2.86 \times 10^{-2}$ & $2.59 \times 10^{-2}$ \\
\hline 800 & $2.12 \times 10^{-2}$ & $2.13 \times 10^{-2}$ & $2.12 \times 10^{-2}$ & $1.99 \times 10^{-2}$ \\
\hline 900 & $1.60 \times 10^{-2}$ & $1.60 \times 10^{-2}$ & $1.60 \times 10^{-2}$ & $1.53 \times 10^{-2}$ \\
\hline 1000 & $1.22 \times 10^{-2}$ & $1.22 \times 10^{-2}$ & $1.22 \times 10^{-2}$ & $1.19 \times 10^{-2}$ \\
\hline \multicolumn{5}{|c|}{$p p \rightarrow b g+X$} \\
\hline 500 & $8.13 \times 10^{0}$ & $8.13 \times 10^{0}$ & $7.93 \times 10^{0}$ & $5.96 \times 10^{0}$ \\
\hline 600 & $5.59 \times 10^{0}$ & $5.59 \times 10^{0}$ & $5.53 \times 10^{0}$ & $4.88 \times 10^{0}$ \\
\hline 700 & $3.98 \times 10^{0}$ & $3.98 \times 10^{0}$ & $3.96 \times 10^{0}$ & $3.73 \times 10^{0}$ \\
\hline 800 & $2.91 \times 10^{0}$ & $2.91 \times 10^{0}$ & $2.90 \times 10^{0}$ & $2.81 \times 10^{0}$ \\
\hline 900 & $2.16 \times 10^{0}$ & $2.16 \times 10^{0}$ & $2.16 \times 10^{0}$ & $2.14 \times 10^{0}$ \\
\hline 1000 & $1.64 \times 10^{0}$ & $1.63 \times 10^{0}$ & $1.63 \times 10^{0}$ & $1.62 \times 10^{0}$ \\
\hline \multicolumn{5}{|c|}{$p p \rightarrow b Z+X$} \\
\hline 500 & $7.87 \times 10^{-1}$ & $7.81 \times 10^{-1}$ & $7.50 \times 10^{-1}$ & $4.79 \times 10^{-1}$ \\
\hline 600 & $5.48 \times 10^{-1}$ & $5.48 \times 10^{-1}$ & $5.31 \times 10^{-1}$ & $4.27 \times 10^{-1}$ \\
\hline 700 & $3.95 \times 10^{-1}$ & $3.94 \times 10^{-1}$ & $3.86 \times 10^{-1}$ & $3.39 \times 10^{-1}$ \\
\hline 800 & $2.92 \times 10^{-1}$ & $2.91 \times 10^{-1}$ & $2.86 \times 10^{-1}$ & $2.61 \times 10^{-1}$ \\
\hline 900 & $2.18 \times 10^{-1}$ & $2.18 \times 10^{-1}$ & $2.15 \times 10^{-1}$ & $2.02 \times 10^{-1}$ \\
\hline 1000 & $1.66 \times 10^{-1}$ & $1.66 \times 10^{-1}$ & $1.64 \times 10^{-1}$ & $1.56 \times 10^{-1}$ \\
\hline
\end{tabular}

We have obtained the cross sections by using the pseudorapidity cuts $\left|\eta_{j, \gamma}\right|<2.5$ and transverse momentum cuts $p_{T}^{j, \gamma}>20-200 \mathrm{GeV}$ for jets and photon, in Table 11 (Tables 12 and 13) for PI (PII, PIII) parametrizations, respectively. Invariant mass distribution of the $b V$ (where $V=\gamma, g$, and $Z$ ) system is shown in Figure 12 for PI parametrization of the signal with $\kappa / \Lambda=0.2 \mathrm{TeV}^{-1}$ and $m_{b^{\prime}}=700 \mathrm{GeV}$ at the center of mass energy $\sqrt{s}=13 \mathrm{TeV}$. It appears from signal significance calculation that the optimized transverse momentum cut is $p_{T}>200 \mathrm{GeV}$ for $b^{\prime}$ analyses.

The backgrounds for the final state $b(\bar{b}) V$ (where $V \equiv$ photon, jet, and $Z$ boson) are given in Table 14. We apply the following cuts to the final state photon and jets as $\left|\eta_{j, \gamma}\right|<2.5$ and $p_{T}^{j, \gamma}>20-200 \mathrm{GeV}$. It can be noted that the background cross section decreases as the $p_{T}$ cuts increase. We assume the efficiency for $b$-tagging to be $\varepsilon_{b}=50 \%$ and the rejection ratios to be $10 \%$ for $c(\bar{c})$ quark jets and $1 \%$ for light quark jets.

In order to reach $3 \sigma$ significance for the signal of $b^{\prime}$ anomalous interactions the required integrated luminosity is shown in Figures 13-15 for parametrizations PI, PII, and PIII at the LHC with $\sqrt{s}=13 \mathrm{TeV}$. The channel $b^{\prime} \rightarrow b \gamma$ requires more integrated luminosity than the other channels. By requiring the signal significance $S S=3$, the contour plots of $\kappa / \Lambda$ and mass of $b^{\prime}$ quark are presented in Figure 16. The results show that one can discover the $b^{\prime}$ quark anomalous couplings down to 0.1 in the $b g$ channel for $m_{b^{\prime}}=500 \mathrm{GeV}$.

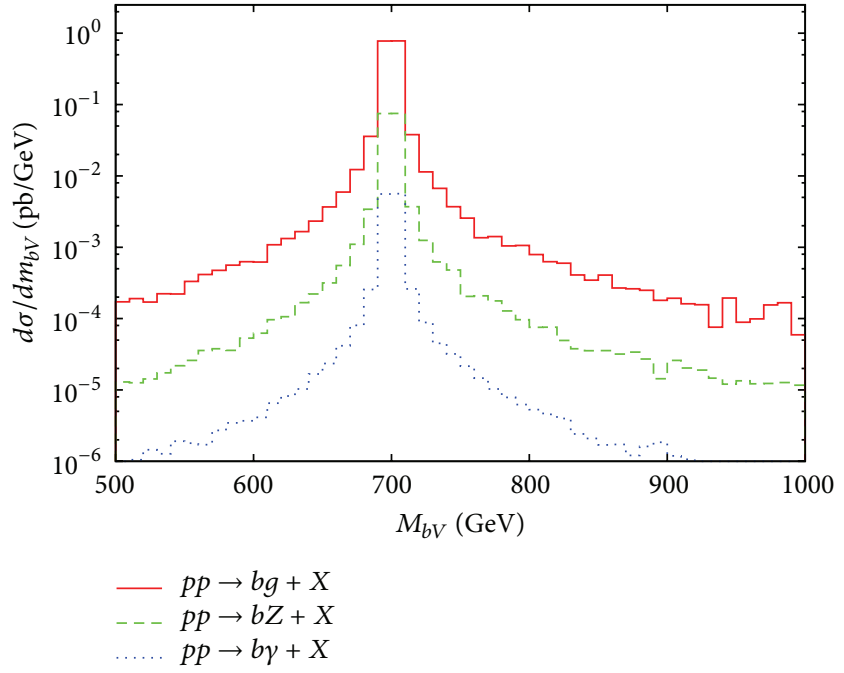

FIGURE 12: Invariant mass distribution of the $b V$ (where $V=\gamma, g$, and $Z$ ) system is shown in Figure 5 for PI parametrization of the signal with $\kappa / \Lambda=0.2 \mathrm{TeV}^{-1}$ and $m_{b^{\prime}}=700 \mathrm{GeV}$ at the center of mass energy $\sqrt{s}=13 \mathrm{TeV}$.

4.2.1. Simulation for $b^{\prime}$ Signal. In the simulation, we have generated $b V$ (where $V=\gamma, g$, and $Z$ ) events for each subprocess and these events are simulated using generic detector 
TABLE 12: The same as Table 11, but for parametrization PII.

\begin{tabular}{|c|c|c|c|c|}
\hline \multirow{2}{*}{$\begin{array}{l}\text { Signal } \\
\text { Mass }(\mathrm{GeV})\end{array}$} & \multicolumn{4}{|c|}{ PII } \\
\hline & $p_{T}>20 \mathrm{GeV}$ & $p_{T}>50 \mathrm{GeV}$ & $p_{T}>100 \mathrm{GeV}$ & $p_{T}>200 \mathrm{GeV}$ \\
\hline \multicolumn{5}{|c|}{$p p \rightarrow b \gamma+X$} \\
\hline 500 & $5.18 \times 10^{-3}$ & $5.26 \times 10^{-3}$ & $5.04 \times 10^{-3}$ & $3.54 \times 10^{-3}$ \\
\hline 600 & $3.38 \times 10^{-3}$ & $3.37 \times 10^{-3}$ & $3.36 \times 10^{-3}$ & $2.77 \times 10^{-3}$ \\
\hline 700 & $2.32 \times 10^{-3}$ & $2.31 \times 10^{-3}$ & $2.30 \times 10^{-3}$ & $2.05 \times 10^{-3}$ \\
\hline 800 & $1.71 \times 10^{-3}$ & $1.63 \times 10^{-3}$ & $1.64 \times 10^{-3}$ & $1.50 \times 10^{-3}$ \\
\hline 900 & $1.17 \times 10^{-3}$ & $1.16 \times 10^{-3}$ & $1.17 \times 10^{-3}$ & $1.11 \times 10^{-3}$ \\
\hline 1000 & $8.60 \times 10^{-4}$ & $8.58 \times 10^{-4}$ & $8.55 \times 10^{-4}$ & $8.24 \times 10^{-4}$ \\
\hline \multicolumn{5}{|c|}{$p p \rightarrow b g+X$} \\
\hline 500 & $7.40 \times 10^{-1}$ & $7.39 \times 10^{-1}$ & $7.21 \times 10^{-1}$ & $5.16 \times 10^{-1}$ \\
\hline 600 & $4.83 \times 10^{-1}$ & $4.80 \times 10^{-1}$ & $4.81 \times 10^{-1}$ & $3.98 \times 10^{-1}$ \\
\hline 700 & $3.22 \times 10^{-1}$ & $3.22 \times 10^{-1}$ & $3.20 \times 10^{-1}$ & $2.89 \times 10^{-1}$ \\
\hline 800 & $2.24 \times 10^{-1}$ & $2.21 \times 10^{-1}$ & $2.21 \times 10^{-1}$ & $2.04 \times 10^{-1}$ \\
\hline 900 & $1.5 \times 10^{-1}$ & $1.58 \times 10^{-1}$ & $1.58 \times 10^{-1}$ & $1.49 \times 10^{-1}$ \\
\hline 1000 & $1.14 \times 10^{-1}$ & $1.14 \times 10^{-1}$ & $1.13 \times 10^{-1}$ & $1.10 \times 10^{-1}$ \\
\hline \multicolumn{5}{|c|}{$p p \rightarrow b Z+X$} \\
\hline 500 & $6.89 \times 10^{-2}$ & $6.85 \times 10^{-2}$ & $6.45 \times 10^{-2}$ & $4.23 \times 10^{-2}$ \\
\hline 600 & $4.52 \times 10^{-2}$ & $4.51 \times 10^{-2}$ & $4.34 \times 10^{-2}$ & $3.53 \times 10^{-2}$ \\
\hline 700 & $3.12 \times 10^{-2}$ & $3.11 \times 10^{-2}$ & $3.05 \times 10^{-2}$ & $2.65 \times 10^{-2}$ \\
\hline 800 & $2.19 \times 10^{-2}$ & $2.18 \times 10^{-2}$ & $2.15 \times 10^{-2}$ & $1.95 \times 10^{-2}$ \\
\hline 900 & $1.56 \times 10^{-2}$ & $1.56 \times 10^{-2}$ & $1.55 \times 10^{-2}$ & $1.44 \times 10^{-2}$ \\
\hline 1000 & $1.14 \times 10^{-2}$ & $1.13 \times 10^{-2}$ & $1.13 \times 10^{-2}$ & $1.07 \times 10^{-2}$ \\
\hline
\end{tabular}

TABle 13: The same as Table 11, but for parametrization PIII.

\begin{tabular}{|c|c|c|c|c|}
\hline \multirow{2}{*}{$\begin{array}{l}\text { Signal } \\
\text { Mass }(\mathrm{GeV})\end{array}$} & \multicolumn{4}{|c|}{ PIII } \\
\hline & $p_{T}>20 \mathrm{GeV}$ & $p_{T}>50 \mathrm{GeV}$ & $p_{T}>100 \mathrm{GeV}$ & $p_{T}>200 \mathrm{GeV}$ \\
\hline \multicolumn{5}{|c|}{$p p \rightarrow b \gamma+X$} \\
\hline 500 & $13.1 \times 10^{-2}$ & $13.14 \times 10^{-2}$ & $12.75 \times 10^{-2}$ & $8.92 \times 10^{-2}$ \\
\hline 600 & $8.59 \times 10^{-2}$ & $8.58 \times 10^{-2}$ & $8.44 \times 10^{-2}$ & $7.03 \times 10^{-2}$ \\
\hline 700 & $5.82 \times 10^{-2}$ & $5.82 \times 10^{-2}$ & $5.77 \times 10^{-2}$ & $5.17 \times 10^{-2}$ \\
\hline 800 & $4.07 \times 10^{-2}$ & $4.07 \times 10^{-2}$ & $4.06 \times 10^{-2}$ & $3.77 \times 10^{-2}$ \\
\hline 900 & $2.92 \times 10^{-2}$ & $2.92 \times 10^{-2}$ & $2.91 \times 10^{-2}$ & $2.77 \times 10^{-2}$ \\
\hline 1000 & $2.14 \times 10^{-2}$ & $2.13 \times 10^{-2}$ & $2.13 \times 10^{-2}$ & $2.06 \times 10^{-2}$ \\
\hline \multicolumn{5}{|c|}{$p p \rightarrow b g+X$} \\
\hline 500 & $19.04 \times 10^{0}$ & $18.96 \times 10^{0}$ & $18.43 \times 10^{0}$ & $12.86 \times 10^{0}$ \\
\hline 600 & $12.19 \times 10^{0}$ & $12.13 \times 10^{0}$ & $11.93 \times 10^{0}$ & $9.92 \times 10^{0}$ \\
\hline 700 & $8.08 \times 10^{0}$ & $8.07 \times 10^{0}$ & $8.02 \times 10^{0}$ & $7.17 \times 10^{0}$ \\
\hline 800 & $5.57 \times 10^{0}$ & $5.57 \times 10^{0}$ & $5.55 \times 10^{0}$ & $5.15 \times 10^{0}$ \\
\hline 900 & $3.94 \times 10^{0}$ & $3.94 \times 10^{0}$ & $3.94 \times 10^{0}$ & $3.74 \times 10^{0}$ \\
\hline 1000 & $2.85 \times 10^{0}$ & $2.85 \times 10^{0}$ & $2.85 \times 10^{0}$ & $2.74 \times 10^{0}$ \\
\hline \multicolumn{5}{|c|}{$p p \rightarrow b Z+X$} \\
\hline 500 & $1.76 \times 10^{0}$ & $1.75 \times 10^{0}$ & $1.65 \times 10^{0}$ & $1.05 \times 10^{0}$ \\
\hline 600 & $1.15 \times 10^{0}$ & $1.14 \times 10^{0}$ & $1.11 \times 10^{0}$ & $8.80 \times 10^{-1}$ \\
\hline 700 & $7.83 \times 10^{-1}$ & $7.80 \times 10^{-1}$ & $7.60 \times 10^{-1}$ & $6.61 \times 10^{-1}$ \\
\hline 800 & $5.47 \times 10^{-1}$ & $5.41 \times 10^{-1}$ & $5.31 \times 10^{-1}$ & $4.80 \times 10^{-1}$ \\
\hline 900 & $3.92 \times 10^{-1}$ & $3.90 \times 10^{-1}$ & $3.82 \times 10^{-1}$ & $3.60 \times 10^{-1}$ \\
\hline 1000 & $2.86 \times 10^{-1}$ & $2.82 \times 10^{-1}$ & $2.80 \times 10^{-1}$ & $2.62 \times 10^{-1}$ \\
\hline
\end{tabular}


TABLE 14: The cross sections (in pb) for the backgrounds $\left(b(\bar{b}) V, c(\bar{c}) V\right.$, and $j V$, where $V=$ photon, jet, and $Z$ boson) with $p_{T}$ cuts on the jets and photon at the center of mass energy $\sqrt{s}=13 \mathrm{TeV}$.

\begin{tabular}{|c|c|c|c|c|}
\hline Background & $p_{T}>20 \mathrm{GeV}$ & $p_{T}>50 \mathrm{GeV}$ & $p_{T}>100 \mathrm{GeV}$ & $p_{T}>200 \mathrm{GeV}$ \\
\hline$p p \rightarrow b(\bar{b}) \gamma+X$ & $2.99 \times 10^{3}$ & $1.35 \times 10^{2}$ & $9.04 \times 10^{0}$ & $4.02 \times 10^{-1}$ \\
\hline$p p \rightarrow c(\bar{c}) \gamma+X$ & $1.87 \times 10^{4}$ & $8.15 \times 10^{2}$ & $5.40 \times 10^{1}$ & $2.43 \times 10^{0}$ \\
\hline$p p \rightarrow j \gamma+X$ & $5.43 \times 10^{4}$ & $3.27 \times 10^{3}$ & $3.38 \times 10^{2}$ & $2.85 \times 10^{1}$ \\
\hline$p p \rightarrow b(\bar{b}) j+X$ & $7.83 \times 10^{6}$ & $3.05 \times 10^{5}$ & $1.92 \times 10^{4}$ & $8.93 \times 10^{2}$ \\
\hline$p p \rightarrow c(\bar{c}) j+X$ & $1.22 \times 10^{7}$ & $4.55 \times 10^{5}$ & $2.89 \times 10^{4}$ & $1.35 \times 10^{3}$ \\
\hline$p p \rightarrow j j+X$ & $2.43 \times 10^{8}$ & $8.54 \times 10^{6}$ & $5.44 \times 10^{5}$ & $2.80 \times 10^{4}$ \\
\hline$p p \rightarrow b(\bar{b}) Z+X$ & $5.02 \times 10^{2}$ & $1.35 \times 10^{2}$ & $2.25 \times 10^{1}$ & $1.56 \times 10^{0}$ \\
\hline$p p \rightarrow c(\bar{c}) Z+X$ & $5.96 \times 10^{2}$ & $1.58 \times 10^{2}$ & $2.64 \times 10^{1}$ & $1.83 \times 10^{0}$ \\
\hline$p p \rightarrow j Z+X$ & $8.00 \times 10^{3}$ & $2.08 \times 10^{3}$ & $4.08 \times 10^{2}$ & $4.12 \times 10^{1}$ \\
\hline
\end{tabular}

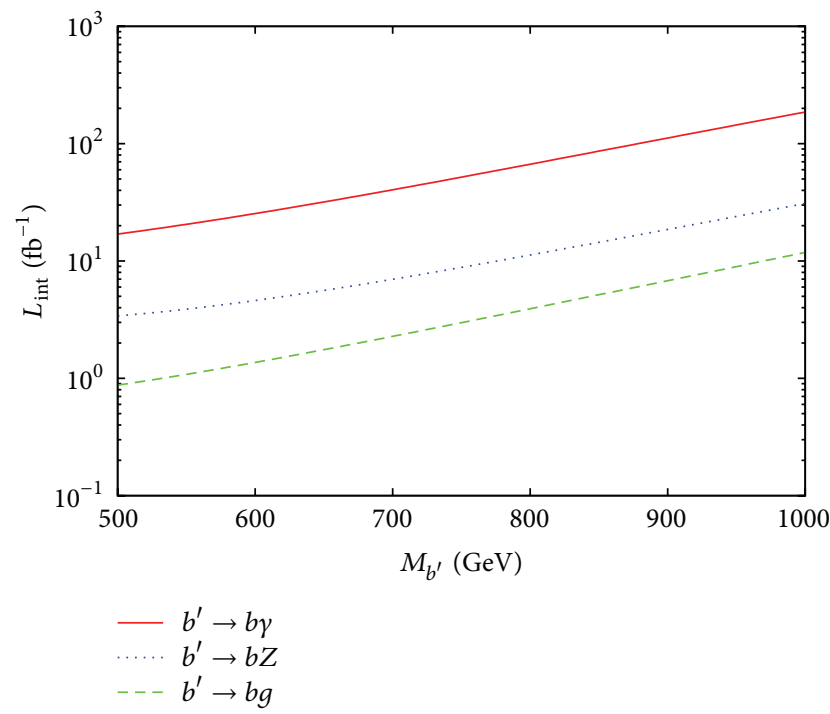

FIGURE 13: Integrated luminosity required to reach $3 \sigma$ significance for the signal of $b^{\prime}$ anomalous interactions for parametrization PI at the LHC with $\sqrt{s}=13 \mathrm{TeV}$.

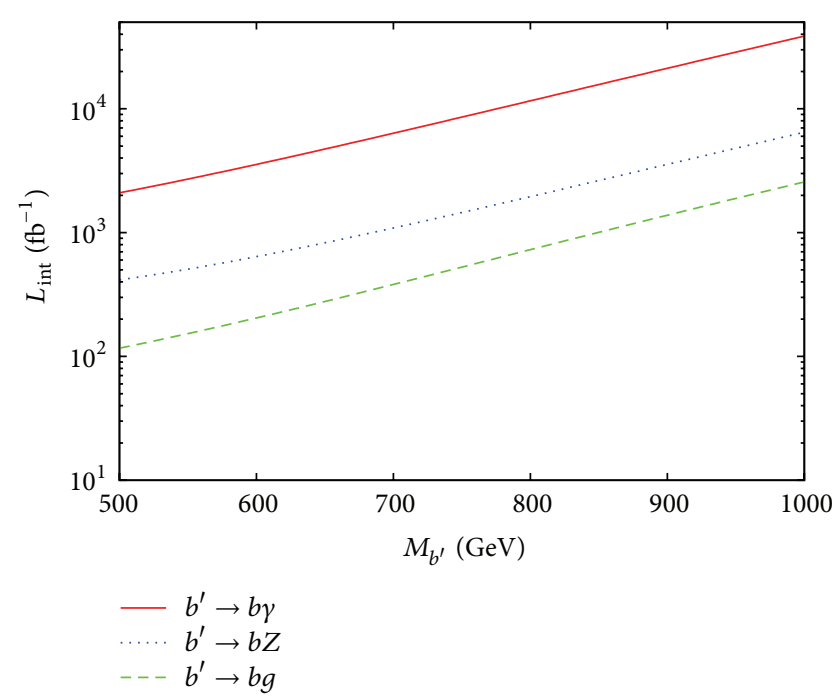

FIgURE 14: The same as Figure 13, but for parametrization PII.

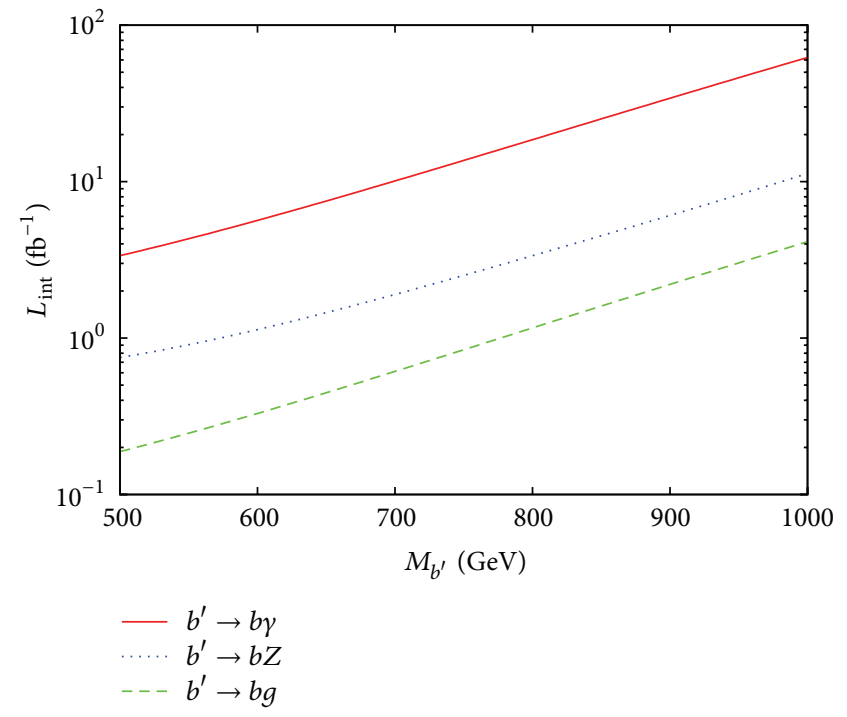

FIgURE 15: The same as Figure 13, but for parametrization PIII.

parameters to include detector effects such as tracking, tagging efficiencies, and smearing effects. After the simulation, the typical kinematical distributions are shown in Figures 1718.

In the analysis, the signal (with $\kappa / \Lambda=0.3 \mathrm{TeV}^{-1}$ and $m_{b^{\prime}}=700 \mathrm{GeV}$ ) and the corresponding background are taken into account. The invariant mass of the new heavy quark $b^{\prime}$ can be reconstructed from a $b_{\text {jet }}$ and a neutral gauge boson (where the $Z$ boson can also be reconstructed from its dilepton or hadronic decay). For the $b \gamma$ production, we require a large $p_{T}^{\gamma}(>100 \mathrm{GeV})$ for photon and large $p_{T}^{j}$ $(>100 \mathrm{GeV})$ for jet and pseudorapidity $\left|\eta^{j, \gamma}\right|(<2.5)$. For the $b \gamma$ signal channel, the invariant mass distributions for signal and background events are shown in Figure 19. The large $p_{T}^{j, \gamma}$ and the requirement of single $b$-tagging allow a better separation of the signal (for $b \gamma$ channel) from the background, and then we find a precise limit for the anomalous coupling in this channel. For the $b g$ and $b Z$ production, we require two high $p_{T}$ jets (one $b$-jet) and a high $p_{T}$ jet in addition to the reconstructed mass $m_{Z}^{\text {rec }}$, respectively. The main character of 


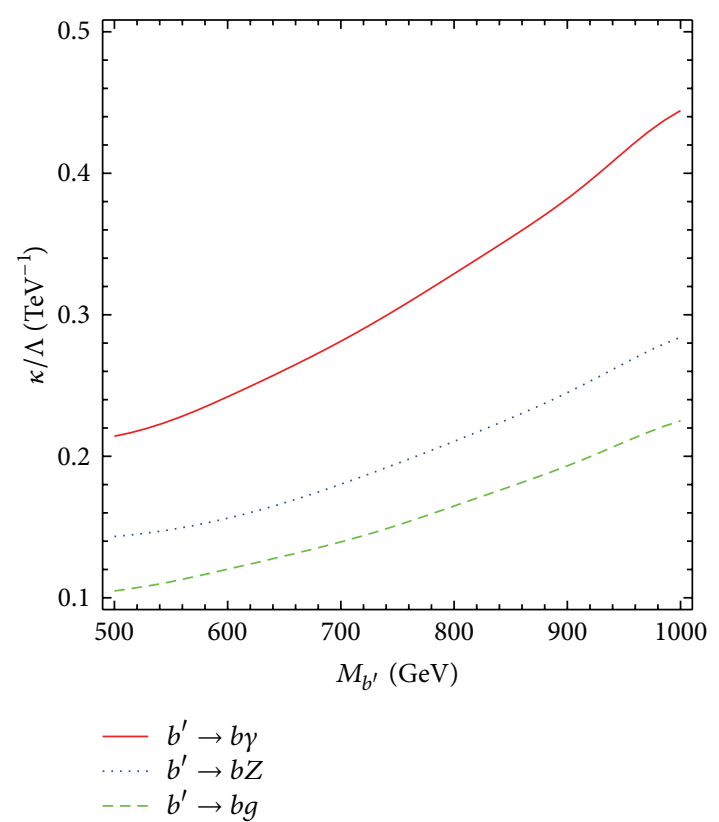

FIGURE 16: The contour plot of anomalous coupling and mass of new heavy quark $b^{\prime}$ for the dynamical parametrization explained in the text with a significance of $3 \sigma$ at $\sqrt{s}=13 \mathrm{TeV}$ and $L_{\text {int }}=100 \mathrm{fb}^{-1}$.

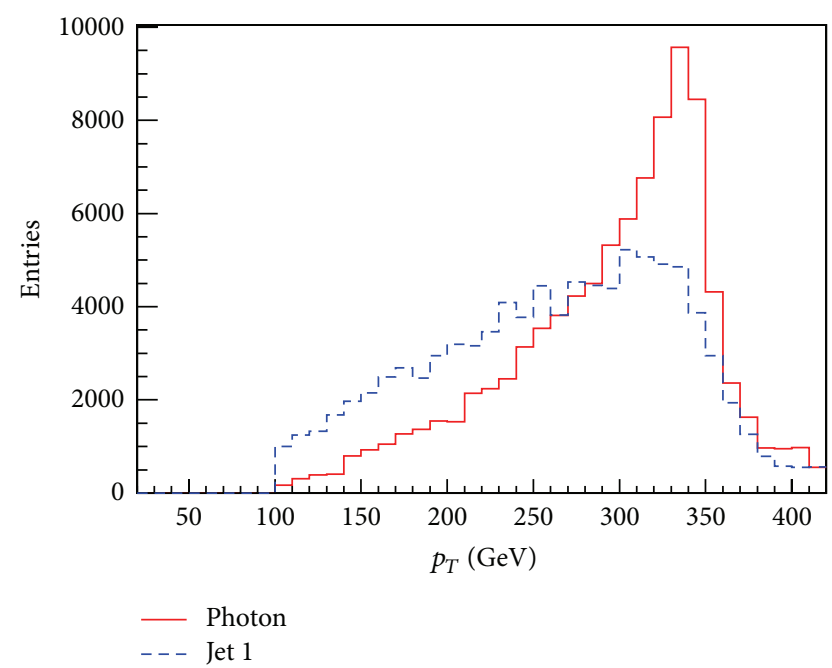

FIgURE 17: Transverse momentum distributions of leading jet and photon ( $b \gamma$ production) for signal after detector simulation.

the signal is the high $p_{T}^{j}$ and/or $p_{T}^{\gamma}$ and single $b$-tagged jet. We calculate the signal and background events in the range $\left|m_{b^{\prime}}^{\text {rec }}(\mathrm{GeV})-700 \mathrm{GeV}\right|<50 \mathrm{GeV}$ and we find a similar significance as shown in Figure 16.

\section{Conclusion}

The new heavy quarks of up-type and down-type can be produced with large numbers at the LHC if they have the anomalous couplings (via flavor changing neutral current) that well dominate over the charged current interactions. The

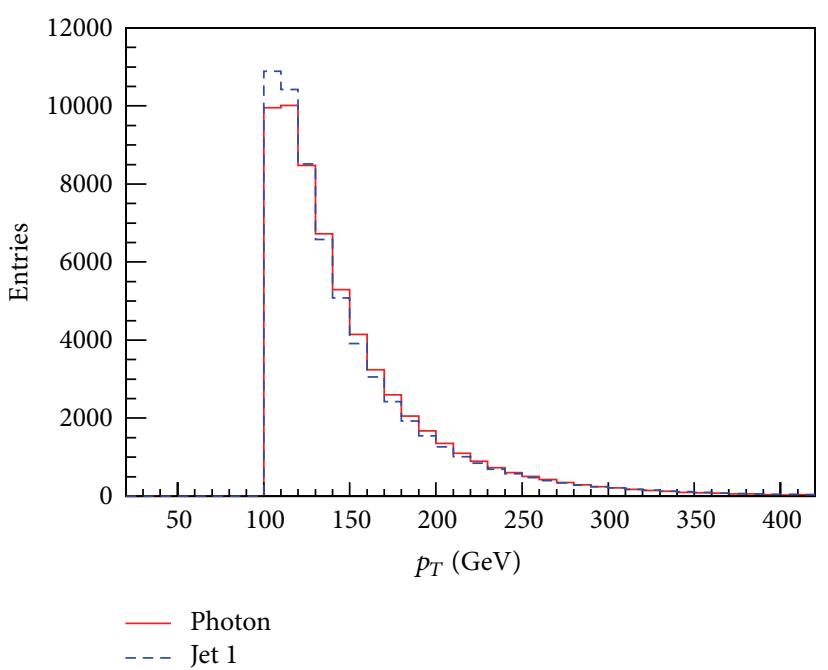

Figure 18: Transverse momentum distributions of leading jet and photon ( $j \gamma$ production) for background at the given conditions mentioned in the text.

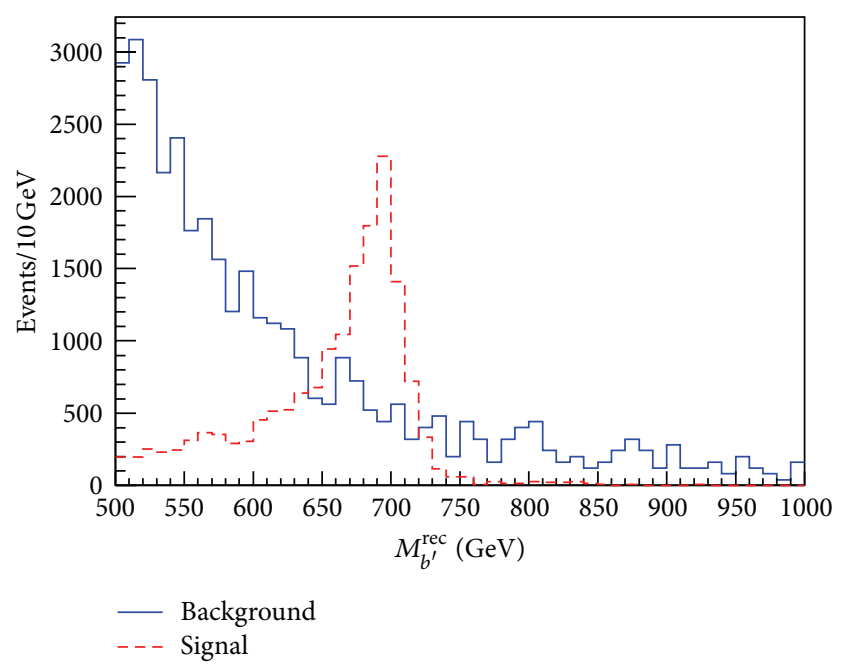

FIGURE 19: The reconstructed mass distributions for background and signal $(b \gamma)$ with $m_{b^{\prime}}=700 \mathrm{GeV}$ and $\kappa / \Lambda=0.3 \mathrm{TeV}^{-1}$.

single production of new heavy quarks can be achieved through the anomalous interactions at the LHC with $\sqrt{s}=$ $13 \mathrm{TeV}$. The anomalous vertices could appear significantly at leading order processes due to the possibility of new heavy quarks. From the results of signal significance calculations for $t^{\prime}\left(b^{\prime}\right)$ anomalous productions, the sensitivity to the anomalous couplings $\kappa^{t^{\prime}} / \Lambda\left(\kappa^{b^{\prime}} / \Lambda\right)$ can be reached down to $0.10 \mathrm{TeV}^{-1}\left(0.15 \mathrm{TeV}^{-1}\right)$ in the lepton $+b_{\text {jet }}+$ jet $+\operatorname{MET}\left(b_{\text {jet }}+\right.$ jet) channel at $\sqrt{s}=13 \mathrm{TeV}$, assuming a dynamical parametrization for the anomalous couplings and the mass of 750 $\mathrm{GeV}$ for the new heavy quarks. The observability limits on the anomalous couplings obtained after the simulation are comparable with the partonic level analysis in the photon and $Z$ boson associated channels, whereas the productions $t g$ and $b g$ are less comparable due to the fast simulation method. In 
any case the single $b$ tagging will play an important role in probing new heavy quarks and reducing the background.

\section{Conflict of Interests}

The authors declare that there is no conflict of interests regarding the publication of this paper.

\section{Acknowledgment}

This work was supported in part by Turkish Atomic Energy Authority (TAEA) under Project Grant no. 2011TAEKCERNA5.H2.P1.01-19.

\section{References}

[1] H. J. He, N. Polonsky, and S. F. Su, "Extra families, Higgs spectrum, and oblique corrections," Physical Review D, vol. 64, no. 5, Article ID 053004, 11 pages, 2001.

[2] B. Holdom, W. S. Hou, T. Hurth, M. L. Mangano, S. Sultansoy, and G. Ünel, "Four statements about the fourth generation," PMC Physics A, vol. 3, article 4, 2009.

[3] A. Atre, M. Carena, T. Han, and J. Santiago, "Heavy quarks above the top at the Tevatron," Physical Review D, vol. 79, Article ID 054018, 2009.

[4] A. Atre, G. Azuelos, M. Carena et al., "Model-independent searches for new quarks at the LHC," Journal of High Energy Physics, vol. 2011, no. 8, article 080, 2011.

[5] N. Chen and H. J. He, "LHC signatures of two-Higgs-doublets with fourth family," Journal of High Energy Physics, vol. 2012, article 062, 2012.

[6] M. S. Chanowitz, "Electroweak constraints on the fourth generation at two loop order," Physical Review D, vol. 88, Article ID 015012, 2013.

[7] S. Chakdar, K. Ghosh, S. Nandi, and S. K. Rai, "Collider signatures of mirror fermions in the framework of a left-right mirror model," Physical Review D, vol. 88, Article ID 095005, 2013.

[8] X. F. Wang, C. Du, and H. J. He, "LHC Higgs signatures from topflavor seesaw mechanism," Physics Letters B, vol. 723, no. 45, pp. 314-323, 2013.

[9] S. Bar-Shalom, M. Geller, S. Nandi, and A. Soni, "Two higgs doublets, a 4th generation and a $125 \mathrm{GeV}$ higgs: a review," Advances in High Energy Physics, vol. 2013, Article ID 672972, 28 pages, 2013.

[10] H. Fritzsch and D. Holtmannspötter, "The production of single t-quarks at LEP and HERA," Physics Letters B, vol. 457, no. 1-3, pp. 186-192, 1999.

[11] G. Aad, B. Abbott, J. Abdallah et al., "Search for down-type fourth generation quarks with the ATLAS detector in events with one lepton and hadronically decaying $W$ bosons," Physical Review Letters, vol. 109, Article ID 032001, 2012.

[12] S. Chatrchyan, V. Khachatryan, A. M. Sirunyan et al., "Search for pair produced fourth-generation up-type quarks in pp collisions at $\sqrt{s}=7 \mathrm{TeV}$ with a lepton in the final state," Physics Letters B, vol. 718, pp. 307-328, 2012.

[13] G. Aad, B. Abbott, J. Abdallah et al., "Search for pair and single production of new heavy quarks that decay to a $Z$ boson and a third-generation quark in $p p$ collisions at $\sqrt{s}=8 \mathrm{TeV}$ with the ATLAS detector," Journal of High Energy Physics, vol. 2014, no. 11, article 104, 2014.
[14] G. Aad, T. Abajyan, B. Abbott et al., "Search for pair production of heavy top-like quarks decaying to a high-pTW boson and a $b$ quark in the lepton plus jets final state at $\sqrt{s}=7 \mathrm{TeV}$ with the ATLAS detector," Physics Letters B, vol. 718, no. 4-5, pp. 12841302, 2013.

[15] S. Chatrchyan, V. Khachatryan, A. M. Sirunyan et al., "Search for heavy, top-like quark pair production in the dilepton final state in pp collisions at $\sqrt{s}=7 \mathrm{TeV}$," Physics Letters B, vol. 716, no. 1, pp. 103-121, 2012.

[16] G. Aad, B. Abbott, J. Abdallah et al., "Search for heavy vectorlike quarks coupling to light quarks in proton-proton collisions at $\sqrt{s}=7$ Tev with the ATLAS detector," Physics Letters B, vol. 712, no. 1-2, pp. 22-39, 2012.

[17] G. Aad, T. Abajyan, B. Abbott et al., "Search for a heavy topquark partner in final states with two leptons with the ATLAS detector at the LHC," Journal of High Energy Physics, vol. 2012, article 94, 2012.

[18] S. Chatrchyan, V. Khachatryan, A. M. Sirunyan et al., "Combined search for the quarks of a sequential fourth generation," Physical Review D, vol. 86, no. 11, Article ID 112003, 20 pages, 2012.

[19] R. Ciftci, "Anomalous single production of the fourth generation quarks at the CERN LHC," Physical Review D, vol. 78, Article ID 075018, 2008.

[20] I. T. Çakır, H. D. Yıldız, O. Çakır, and G. Ünel, "Anomalous resonant production of the fourth-family up-type quarks at the LHC," Physical Review D, vol. 80, Article ID 095009, 2009.

[21] M. Sahin, S. Sultansoy, and S. Turkoz, "Searching for the fourth family quarks through anomalous decays," Physical Review D, vol. 82, no. 5, Article ID 051503, 2010.

[22] M. Bobrowski, A. Lenz, J. Riedl, and J. Rohrwild, "How much space is left for a new family of fermions?" Physical Review D, vol. 79, no. 11, Article ID 113006, 15 pages, 2009.

[23] G. Eilam, B. Melić, and J. Trampetić, " $C P$ violation and the fourth generation," Physical Review D, vol. 80, no. 11, Article ID 116003, 2009.

[24] O. Cobanoglu, E. Ozcan, S. Sultansoy, and G. Ünel, “OPUCEM: a library with error checking mechanism for computing oblique parameters," Computer Physics Communications, vol. 182, no. 8, pp. 1732-1743, 2011.

[25] T. Han and J. L. Hewett, "Top-charm associated production in high energy $e^{+} e^{-}$collisions," Physical Review D, vol. 60, Article ID 074015, 1999.

[26] A. Belyaev, N. D. Christensen, and A. Pukhov, "CalcHEP 3.4 for collider physics within and beyond the standard model," Computer Physics Communications, vol. 184, no. 7, pp. 17291769, 2013.

[27] J. Pumplin, D. Robert Stump, J. Huston, H.-L. Lai, P. Nadolsky, and W.-K. Tung, "New generation of Parton distributions with uncertainties from global QCD analysis," Journal of High Energy Physics, vol. 2002, article 012, 2002.

[28] G. L. Bayatian, S. Chatrchyan, G. Hmayakyan et al., "CMS physics technical design report, volume II: physics performance," Journal of Physics G: Nuclear and Particle Physics, vol. 34, no. 6, p. 995, 2007.

[29] T. Sjöstrand, S. Mrenna, and P. Skands, "PYTHIA 6.4 physics and manual," Journal of High Energy Physics, vol. 2006, no. 5, p. 026, 2006.

[30] J. Conway, R. Culbertson, and R. Demina, Pretty Good Simulation (PGS4), http://www.physics.ucdavis.edu/ conway/ research/software/pgs/pgs4-general.htm. 
[31] EXROOTANALYSIS package for PGS4 data analysis, http://madgraph.hep.uiuc.edu/Downloads/ExRootAnalysis/.

[32] R. Brun et al., An object oriented data analysis framework (ROOT), https://root.cern.ch/drupal/.

[33] F. del Aguila and J. A. Aguilar-Saavedra, "Multilepton production via top flavour-changing neutral couplings at the CERN LHC," Nuclear Physics B, vol. 576, pp. 56-84, 2000.

[34] T. Han, M. Hosch, K. Whisnant, B.-L. Young, and X. Zhang, "Single top quark production via FCNC couplings at hadron colliders," Physical Review D, vol. 58, Article ID 073008, 1998.

[35] T. Stelzer, Z. Sullivan, and S. Willenbrock, "Single-top-quark production at hadron colliders," Physical Review D, vol. 58, no. 9, Article ID 094021, 11 pages, 1998. 

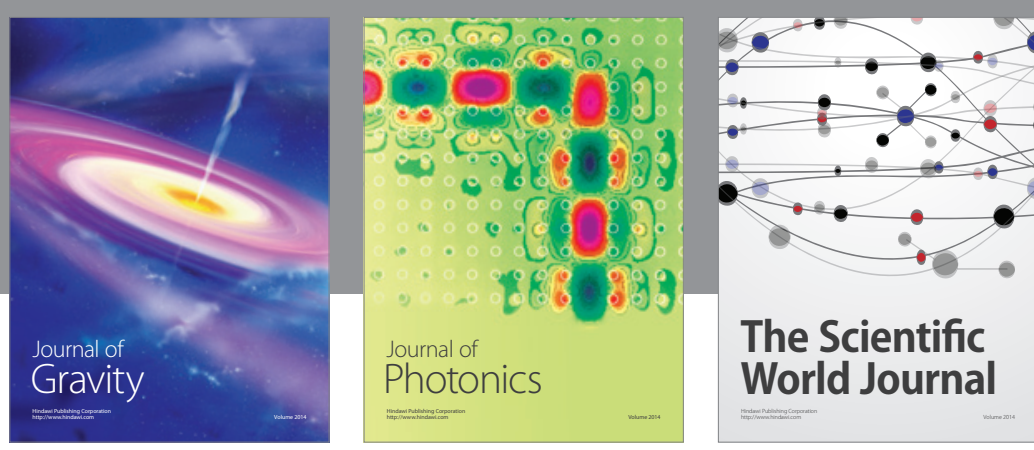

The Scientific World Journal
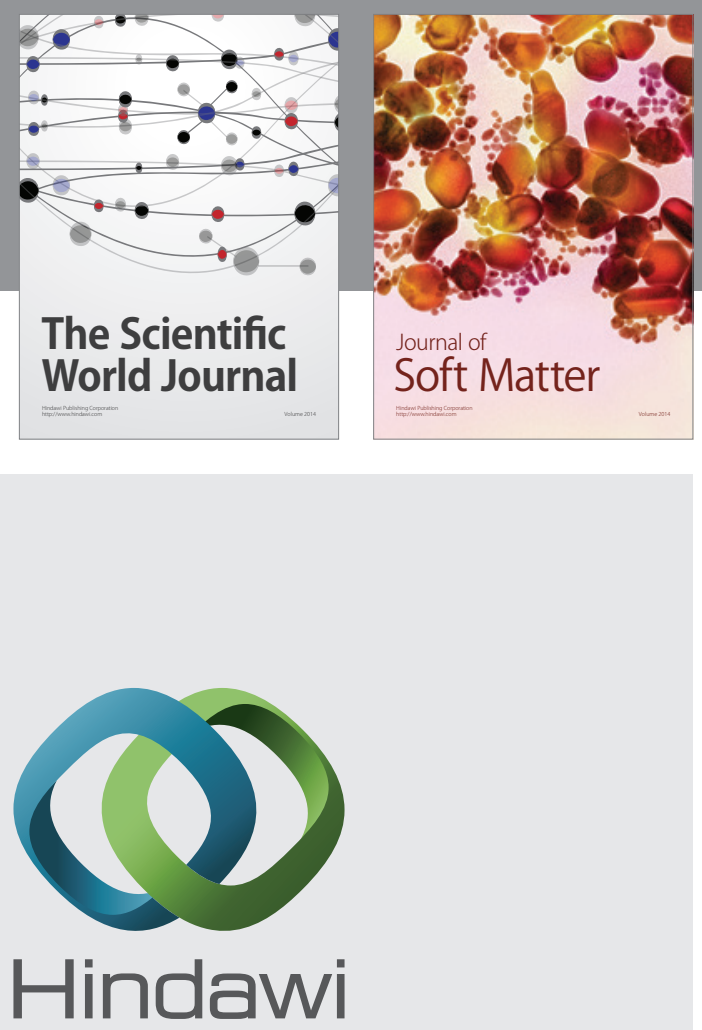

Submit your manuscripts at

http://www.hindawi.com

nternational Journal of

Statistical Mechanics
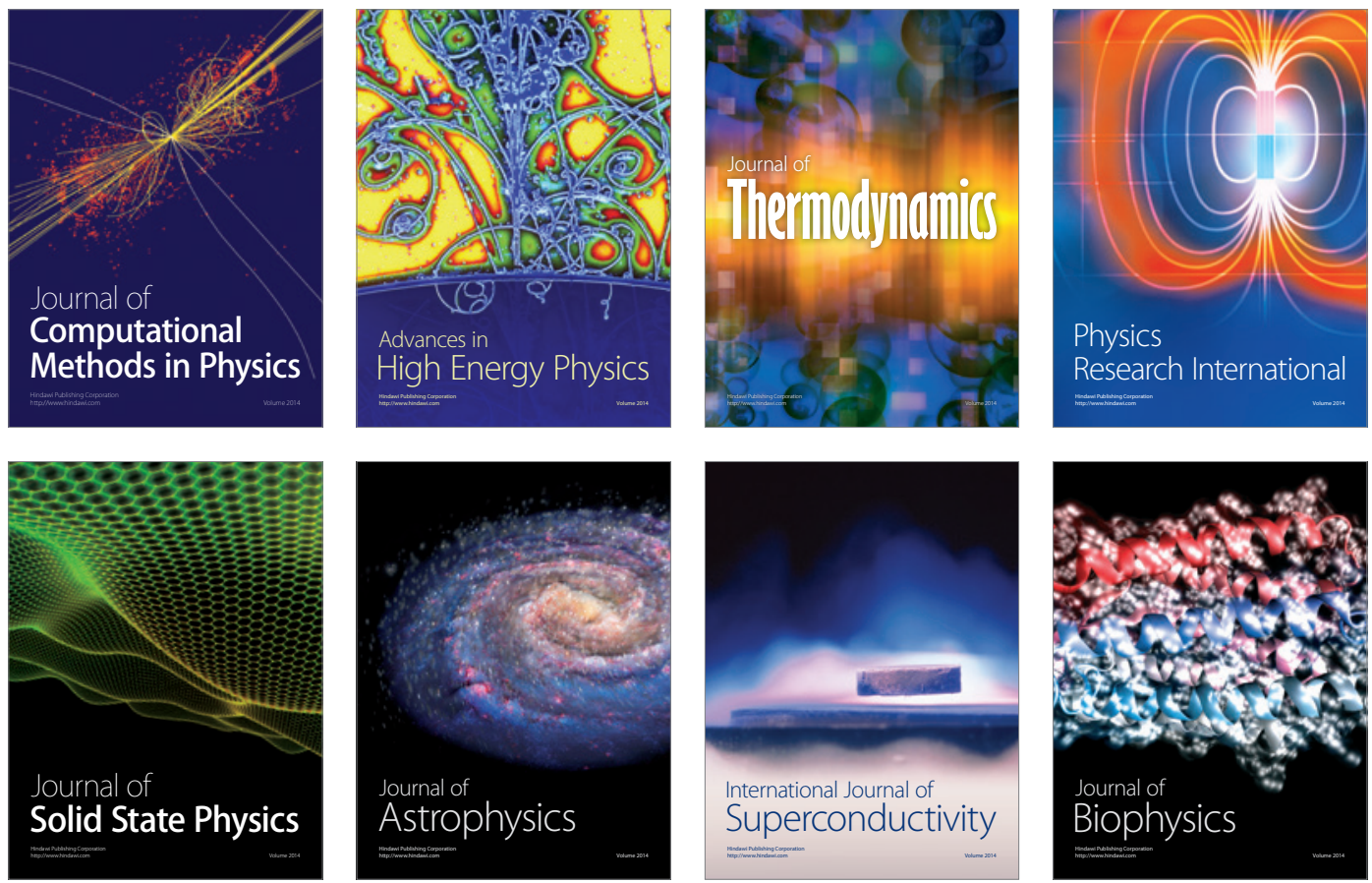
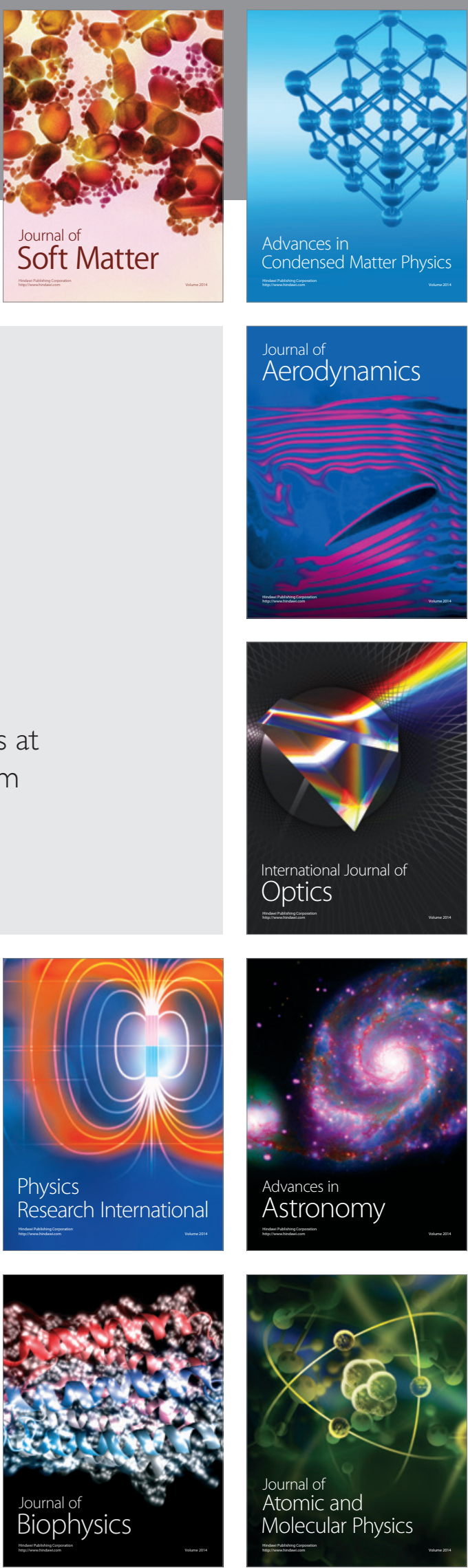